\title{
Theory of Current-Induced Magnetization Precession
}

\author{
M.D. Stiles \\ National Institute of Standards and Technology, Gaithersburg, MD 20899-8412 \\ Jiang Xiao and A. Zangwill \\ School of Physics, Georgia Institute of Technology, Atlanta, GA 30332-0430
}

(Dated: October 29, 2018)

\begin{abstract}
We solve appropriate drift-diffusion and Landau-Lifshitz-Gilbert equations to demonstrate that unpolarized current flow from a non-magnet into a ferromagnet can produce a precession-type instability of the magnetization. The fundamental origin of the instability is the difference in conductivity between majority spins and minority spins in the ferromagnet. This leads to spin accumulation and spin currents that carry angular momentum across the interface. The component of this angular momentum perpendicular to the magnetization drives precessional motion that is opposed by Gilbert damping. Neglecting magnetic anisotropy and magnetostatics, our approximate analytic and exact numerical solutions using realistic values for the material parameters show (for both semi-infinite and thin film geometries) that a linear instability occurs when both the current density and the excitation wave vector parallel to the interface are neither too small nor too large. For many aspects of the problem, the variation of the magnetization in the direction of the current flows makes an important contribution.
\end{abstract}

\section{INTRODUCTION}

Recently, the phenomenon of giant magnetoresistance has been used to unambiguously detect current-induced magnetization reversal in thin magnetic heterostructures. ${ }^{1,2.3}$ These observations confirm theoretical predictions 4.5 that angular momentum can be transferred from current-carrying electrons to the magnetization of a ferromagnetic film. Earlier reports 6 of the observation of this so-called spin-transfer effect attributed a magnetic origin (magnetization reversal, free precession, or spin wave excitation) to anomalies seen in transport measurements. All these experiments used a ferromagnet/non-magnet/ferromagnet spin valve geometry because a "polarizer" ferromagnet was deemed necessary to ensure that the electrons incident on the "analyzer" ferromagnet from the non-magnet would carry a spin-polarized current. Now, two new experimental facts have appeared that bear directly on these issues. First, current injection into a single ferromagnetic layer has been observed to produce resistance anomalies very similar to those seen in the spin valves ${ }^{7}$ Second, microwave emission has been detected in at least some spin valve samples when spin-transfer resistance anomalies occur $\stackrel{8}{6}$

Based on the foregoing, it is natural to inquire whether magnetic precession occurs when an unpolarized current flows (perpendicular to the interface) from a nonmagnetic metal into a ferromagnetic metal. We investigate this question here by performing a linear stability analysis of a presumptive current-induced precessional state. Our approach to this problem of coupled transport and magnetization dynamics derives from previously published work by two of us. One earlier paper ${ }^{9}$ discussed the quantum mechanical origin of spin-transfer torque at a ferromagnetic/non-magnetic interface. Another paper ${ }^{10}$ solved a matrix Boltzmann equation to compute spin currents, spin accumulation, magnetoresis- tance, and spin-transfer torques for multilayers. In the present work, we study both single and multiple interface geometries in the presence of an external magnetic field (normal to the interface) strong enough to saturate the magnetization (Figure 1). Magnetic anisotropy and magnetostatics are neglected for simplicity. Our approximate analytic and exact numerical solutions reveal that an initial instability toward precession occurs when both the current density and the excitation wave vector parallel to the interface are neither too large nor too small. Otherwise, Gilbert damping suppresses the instability. An important feature of our model is that the magnetization is permitted to vary in the direction normal to the interface. Among other things, this means that precession can occur in the film geometry even when the two interfaces are identical. Other systematic features of the precessional state will be described in detail below.

Our results complement and extend previous theoretical studies of current-induced precession in magnetic films. In their pioneering work, Slonczewski ${ }^{4}$ and Berger ${ }^{5}$ used analytic model calculations to predict the critical current for the onset of precession in spin valve structures with uniform but non-collinear magnetizations in the two ferromagnetic layers. Recent work shows that the seemingly different answers they obtained are not inconsistent 11 Two global studies of precession and switching in single-domain spin valves 12 and single-domain particles ${ }^{13}$ supplement the LandauLifshitz-Gilbert equation with the spin-transfer torque derived in Ref. 4. Bazaliy et al ${ }^{14}$ relaxed the assumption of uniform magnetization and studied the effect of a legislated spin current flowing from a non-magnet into a semi-infinite ferromagnet. They found that spontaneous excitation of spin waves occurs when the current density is sufficiently large. Finally, we draw attention to a very recent application 15 of magnetoelectronic circuit theory ${ }^{16}$ to study current-induced precession in an ultra- 


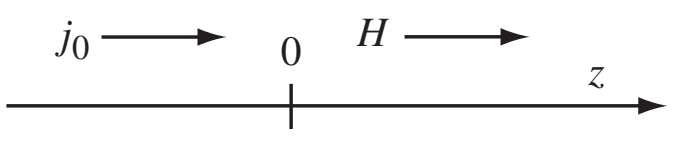

(a)
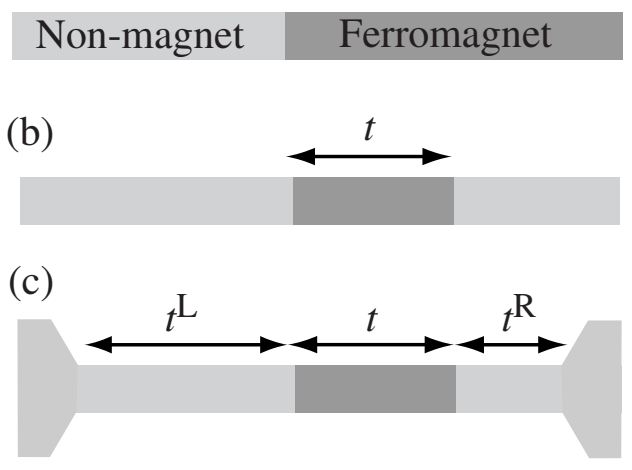

FIG. 1: The external magnetic field and particle current both point in the positive $z$-direction for (a) a single-interface geometry; (b) a thin film geometry; and (c) an electron reservoir geometry. The interfacial areas are infinite in all cases. In (b), the non-magnetic leads are semi-infinite in length. In (c), the non-magnetic leads are attached to electron reservoirs at equilibrium

thin ferromagnetic film with uniform magnetization. For unpolarized current densities above a critical value, the authors predict that unstable spin wave modes exist only for non-zero wave vectors parallel to the interface. Our results confirm this prediction.

The calculations presented in this paper combine phenomenological constitutive equations for the electric current and the spin current (drift-diffusion equations) with a Landau-Lifshitz-Gilbert equation generalized to include spin-transfer torque. The boundary conditions at each non-magnet/ferromagnet interface are chosen so that relevant observables reproduce the results of a Boltzmann equation treatment of the same problem. Using experimental data to fix the parameters, we choose a value for the electric current density, and solve for the interface charge density (chemical potential discontinuity), spin accumulation, and spin current assuming a precessing magnetization with an amplitude and phase that depends on the running variable $z$ in Figure 1 The presumed precession is a legitimate mode of the system if the computed spin-transfer torque balances the ferromagnetic exchange torque at each interface 14 The mode is unstable (spontaneous excitation in the presence of a current) if the imaginary part of the mode frequency is negative. The mode is stable and uninteresting if Gilbert damping overwhelms the effect of spin transfer.

The plan of this paper is as follows. In Section II, we set down the basic definitions and equations of the theory. Section III gives a qualitative picture of the origin of the precessional instability. The boundary conditions for the transport and magnetization equations are given in Section IV. Sections V and VI present the details of the calculation for a single interface geometry and a thin film geometry, respectively. Section VII discusses our results. Section VIII examines the effect of non-magnetic leads using a reservoir geometry $\frac{15}{\underline{15}}$ Section IX discusses some limitations of our model and suggestions for future work. Section $\mathrm{X}$ is a summary. Appendix A derives the effective exchange field for a bounded ferromagnet. The transport boundary equations are derived in Appendix B.

\section{DEFINITIONS \& BASIC EQUATIONS}

In an earlier paper, $\frac{9}{\underline{w}}$ we defined the fundamental variables of spin transport quantum mechanically and described the various relations that exist among them. Here, we proceed phenomenologically and decompose the total number density of conduction electrons into majority and minority contributions $n=n_{\uparrow}+n_{\downarrow}$ so that the current-induced deviations of these quantities from their equilibrium values are related by

$$
\delta n=\delta n_{\uparrow}+\delta n_{\downarrow} .
$$

In the drift-diffusion approximation, the particle current in the non-magnet is

$$
\mathbf{j}^{\mathrm{NM}}=(\sigma / e) \mathbf{E}-D \boldsymbol{\nabla} \delta n,
$$

where $\mathbf{E}$ is the electric field, $\sigma$ is the conductivity, and $D$ is the electron diffusion constant. We speak of spin accumulation in the non-magnet when the spin density

$$
\mathbf{m}=\frac{\hbar}{2}\left(\delta n_{\uparrow}-\delta n_{\downarrow}\right) \hat{\mathbf{m}}
$$

is different from zero. Notice that we use the particle current and the spin density as variables rather than the charge current and the magnetization.

A so-called spin current density

$$
Q_{\alpha \beta}^{\mathrm{NM}}=-D \nabla_{\beta} m_{\alpha}
$$

flows in the non-magnet when the spin accumulation is not uniform in space. In (4), the first index of $Q_{\alpha, \beta}^{\mathrm{NM}}$ labels the $\alpha^{\text {th }}$ Cartesian component of the electron spin vector while the second index labels the $\beta^{\text {th }}$ Cartesian component of the flow direction.

In the ferromagnet, we make explicit use of the fact that there is no appreciable spin accumulation or spin current in the direction perpendicular to the magnetization 9.17 Accordingly, $\delta m=(\hbar / 2)\left(\delta n_{\uparrow}-\delta n_{\downarrow}\right)$ is the spin accumulation so

$$
\mathbf{M}=\left(m_{s}+\delta m\right) \mathbf{u}
$$

is the total spin density. Here, $\mathbf{u}$ is a unit vector, $m_{\mathrm{s}}=$ $\hbar M_{\mathrm{s}} / g \mu_{B}$, and $M_{\mathrm{s}}$ is the saturation magnetization. We adopt a two-current mode ${ }^{18}$ so $\mathbf{j}^{\mathrm{FM}}=\mathbf{j}_{\uparrow}+\mathbf{j}_{\downarrow}$ where

$$
\begin{aligned}
& \mathbf{j}_{\uparrow}=\left(\sigma_{\uparrow} / e\right) \mathbf{E}-D_{\uparrow} \boldsymbol{\nabla} \delta n_{\uparrow} \\
& \mathbf{j}_{\downarrow}=\left(\sigma_{\downarrow} / e\right) \mathbf{E}-D_{\downarrow} \boldsymbol{\nabla} \delta n_{\downarrow},
\end{aligned}
$$


using an obvious notation for the conductivity and diffusion constants of majority and minority spin electrons. Combining all the above, the total particle current in the ferromagnet is

$$
\mathbf{j}^{\mathrm{FM}}=\frac{\sigma_{\uparrow}+\sigma_{\downarrow}}{e} \mathbf{E}-\frac{D_{\uparrow}+D_{\downarrow}}{2} \nabla \delta n-\frac{D_{\uparrow}-D_{\downarrow}}{\hbar} \nabla \delta m .
$$

The corresponding spin current density in the ferromagnet is

$$
Q_{\alpha \beta}^{\mathrm{FM}}=\frac{1}{2} \hbar u_{\alpha}\left(\mathbf{j}_{\uparrow}-\mathbf{j}_{\downarrow}\right)_{\beta} .
$$

Consistent with our neglect of transverse spin accumulation in (5), this formula presumes that the spin part of the spin current in a ferromagnet is always parallel to the spin density. Then, using (6), we get

$$
\begin{aligned}
Q_{\alpha \beta}^{\mathrm{FM}}= & \frac{\hbar}{2} \frac{\sigma_{\uparrow}-\sigma_{\downarrow}}{e} u_{\alpha} E_{\beta}-\frac{\hbar}{2} \frac{D_{\uparrow}-D_{\downarrow}}{2} u_{\alpha} \nabla_{\beta} \delta n \\
& -\frac{D_{\uparrow}+D_{\downarrow}}{2} u_{\alpha} \nabla_{\beta} \delta m .
\end{aligned}
$$

Textbooks show that the charge density inside a current-carrying Ohmic conductor is zero (except within a screening distance of an interface or free surface). Therefore, we are justified to put $\delta n=0$ in (2), (7), and (9). We will also fix the current to be $\mathbf{j}=j_{0} \hat{z}$ everywhere. Using this fact in (7), we can eliminate $\mathbf{E}$ from (9) and simplify the components of the ferromagnetic spin current density that flow in directions longitudinal $(z)$ and transverse $(\perp)$ to the interface normal. The result is

$$
\begin{aligned}
& Q_{\alpha z}^{\mathrm{FM}}=\frac{1}{2} \hbar \gamma^{\mathrm{p}} u_{\alpha} j_{0}-D_{z} u_{\alpha} \nabla_{z} \delta m \\
& Q_{\alpha \perp}^{\mathrm{FM}}=-D_{\perp} u_{\alpha} \nabla_{\perp} \delta m,
\end{aligned}
$$

where $\gamma^{\mathrm{p}}=\left(\sigma_{\uparrow}-\sigma_{\downarrow}\right) /\left(\sigma_{\uparrow}+\sigma_{\downarrow}\right)$ is the degree of current polarization desired by the ferromagnetic bulk, $D_{z}=$ $\left(D_{\uparrow} \sigma_{\downarrow}+D_{\downarrow} \sigma_{\uparrow}\right) /\left(\sigma_{\uparrow}+\sigma_{\downarrow}\right)$ is an effective longitudinal spin diffusion constant, and $D_{\perp}=\frac{1}{2}\left(D_{\uparrow}+D_{\downarrow}\right)$ is an effective transverse spin diffusion constant.

The equation of motion for the spin density in the non-magnet is a continuity equation for the spin accumulation 9 Taking account of distributed spintransfer torque, spin flip scattering and torque from the external magnetic field, we find

$$
\dot{m}_{\alpha}+\nabla_{\beta} Q_{\alpha \beta}^{\mathrm{NM}}+\gamma\left[\mathbf{m} \times \mathbf{H}_{\mathrm{ext}}\right]_{\alpha}+\frac{m_{\alpha}}{\tau_{\mathrm{sf}}}=0
$$

where $\tau_{\text {sf }}$ is the spin-flip scattering time in the nonmagnet and $\gamma \mathbf{H}=\left(g \mu_{B} / \hbar\right) \mathbf{B}$. The equation of motion for the spin density in the ferromagnet is the LandauLifshitz-Gilbert equation supplemented by a term to account for spin-transfer torque as in (11). Specifically,

$$
\dot{M}_{\alpha}+\nabla_{\beta} Q_{\alpha \beta}^{\mathrm{FM}}+\gamma\left[\mathbf{M} \times \mathbf{H}_{\mathrm{eff}}\right]_{\alpha}+\frac{u_{\alpha} \delta m}{\bar{\tau}_{\mathrm{sf}}}=0
$$

where $\bar{\tau}_{\text {sf }}$ is the spin-flip scattering time in the ferromagnet. The effective field $\mathbf{H}_{\text {eff }}$ accounts for the external field, exchange, and damping:

$$
\mathbf{H}_{\mathrm{eff}}=\mathbf{H}_{\mathrm{ext}}+\frac{\omega_{0} l_{\mathrm{ex}}^{2}}{\gamma m_{\mathrm{s}}} \nabla^{2} \mathbf{M}-\frac{\alpha}{\gamma|\mathbf{M}|} \dot{\mathbf{M}} .
$$

Here, $l_{\mathrm{ex}}=\sqrt{2 A_{\mathrm{ex}} / \mu_{0} M_{s}^{2}}$ is the exchange length in a ferromagnet with spin stiffness $A_{\mathrm{ex}}, \omega_{0}=\gamma M_{\mathrm{s}}$, and $\alpha$ is a phenomenological damping parameter. We ignore magnetostatics, but for fields and magnetization perpendicular to the interface, its main effect is to shift the applied magnetic field by an amount $M_{\mathrm{s}}$.

\section{ORIGIN OF THE INSTABILITY}

Two characteristic features of the spin current $Q_{\alpha \beta}$ underlie the qualitative physics of current-induced precession. First, the structure of the spin-transfer torque in (11) and (12) implies that the magnetization at the $z=0$ interface in Figure 1 feels a torque per unit area. It is

$$
\begin{aligned}
\frac{\mathbf{N}_{\mathrm{st}}}{A}=-\lim _{\epsilon \rightarrow 0} \int_{-\epsilon}^{\epsilon} d z \boldsymbol{\nabla} \cdot \mathbf{Q} & =\left(\mathbf{Q}^{\mathrm{NM}}-\mathbf{Q}^{\mathrm{FM}}\right) \cdot \hat{\mathbf{z}} \\
& \simeq \mathbf{Q}_{\perp^{\prime} z}^{\mathrm{NM}}
\end{aligned}
$$

where $\perp^{\prime}$ refers to the directions transverse to the ferromagnetic magnetization $\left(z^{\prime}\right.$ is the direction along the ferromagnetic magnetization). The last line in (14) is true because

$$
Q_{z^{\prime} z}^{\mathrm{NM}}=Q_{z^{\prime} z}^{\mathrm{FM}}
$$

and $Q_{\perp^{\prime} z}^{\mathrm{FM}} \simeq 0$ at the interface between a non-magnet and a ferromagnet due to spin-filtering and other effects, $4,5,9,19$

The second bit of information we need is a linear kinetic equation that relates the transverse spin current at the interface, $\mathbf{Q}_{\perp^{\prime} z}^{\mathrm{NM}}$, to the interfacial densities of our problem (charge accumulation, spin accumulation in the non-magnet, and ferromagnetic spin accumulation). Of these, for a static magnetization only the spin accumulation $\mathbf{m}$ in (3) has a transverse component. Therefore, it must be the case that

$$
\mathbf{Q}_{\perp^{\prime} z}^{\mathrm{NM}}=b \mathbf{m}_{\perp^{\prime}},
$$

where $b$ is a constant. More detailed considerations (see Appendix B) show that $b>0$.

For simplicity, we will study (in this section) a timeindependent situation where electrons flow from a nonmagnet into a ferromagnet with a small amplitude, frozen spin wave at the interface. That is, in (5), we let $\mathbf{u}=$ $\hat{\mathbf{z}}+\mathbf{u}_{\perp} \exp (i \mathbf{K} \cdot \mathbf{R})$ where $\mathbf{R}$ and $\mathbf{K}$ are two-dimensional vectors in the plane of the interface. This means that 


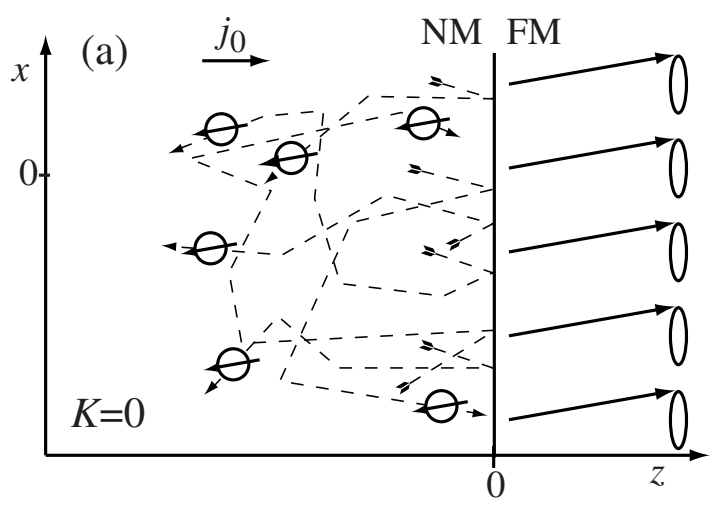

(b)
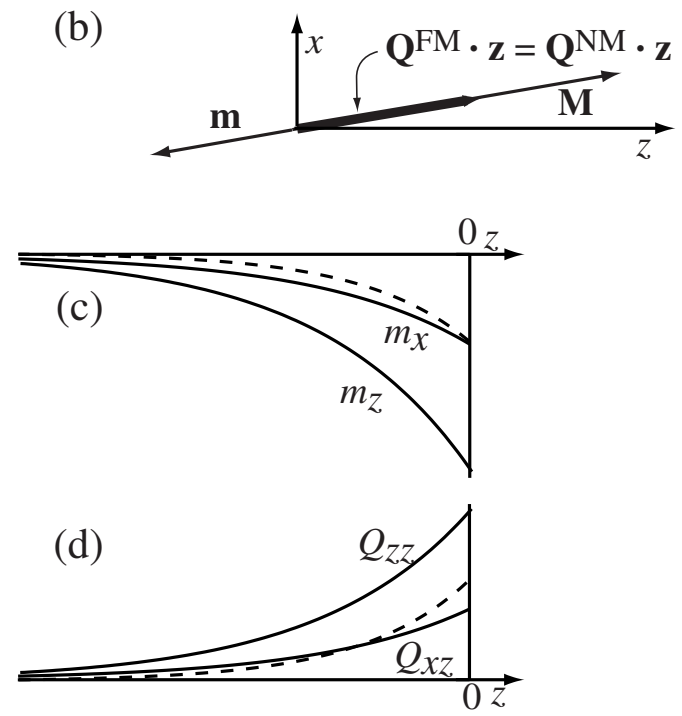

(e)
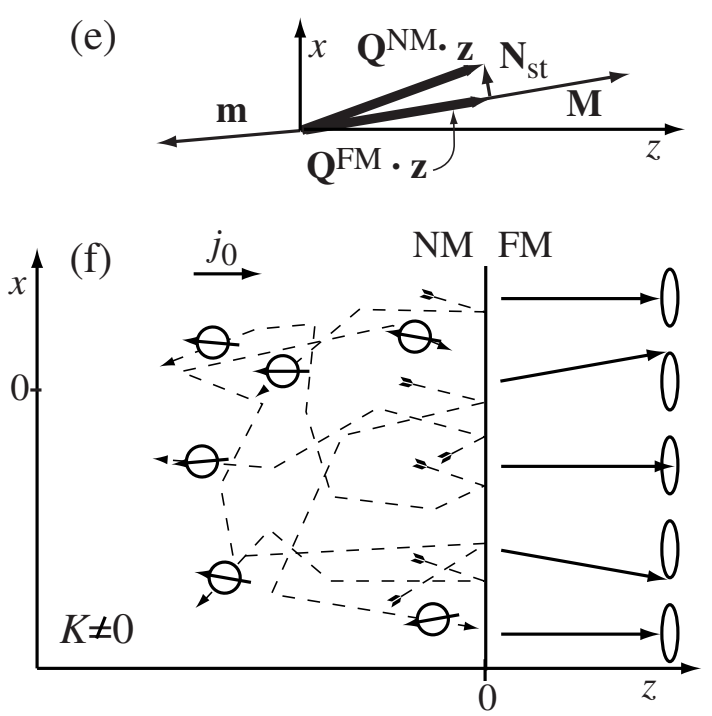

FIG. 2: (a) Cartoon of steady-state spin configuration when $K=0$; (b) spin accumulation and spin current when $K=0$; (c) solid curves: $m_{z}(x=0, z)$ and $m_{x}(x=0, z)$ when $K=0$; dashed curve: $m_{x}(x=0, z)$ when $K \neq 0$; (d) solid curves: $Q_{z z}^{\mathrm{NM}}(x=0, z)$ and $Q_{x z}^{\mathrm{NM}}(x=0, z)$ when $K=0$; dashed curve: $Q_{x z}^{\mathrm{NM}}(x=0, z)$ when $K \neq 0$; (e) same as (b) when $K \neq 0$; (f) same as (a) when $K \neq 0$. See text for details. conduction electrons incident on the ferromagnet from the non-magnet see a static magnetization

$$
\mathbf{M}=M\left\{\operatorname{Re}\left[u_{\perp} e^{i \mathbf{K} \cdot \mathbf{R}}\right], \operatorname{Im}\left[u_{\perp} e^{i \mathbf{K} \cdot \mathbf{R}}\right], 1\right\} .
$$

In the non-magnet itself, (44) and (11) with $\mathbf{H}_{\mathrm{ext}}=0$ combine to give

$$
D \nabla^{2} m_{\alpha}=\frac{m_{\alpha}}{\tau_{\mathrm{sf}}}
$$

for the steady-state spin accumulation in the nonmagnet. This is solved by

$$
\mathbf{m}=\left\{\operatorname{Re}\left[m_{\perp}(z) e^{i \mathbf{K} \cdot \mathbf{R}}\right], \operatorname{Im}\left[m_{\perp}(z) e^{i \mathbf{K} \cdot \mathbf{R}}\right], m_{z}(z)\right\},
$$

if

$$
\begin{aligned}
m_{z}(z) & =m_{z} \exp \left(z / l_{\mathrm{sf}}\right) \\
m_{\perp}(z) & =m_{\perp} \exp (\kappa z)
\end{aligned}
$$

with $l_{\mathrm{sf}}^{2}=D \tau_{\mathrm{sf}}$ and $l_{\mathrm{sf}}^{2} \kappa^{2}=1+l_{\mathrm{sf}}^{2} K^{2}$.

We begin our analysis with the right side of the top panel in Figure 2 This shows a spin pattern in the ferromagnet that is spatially uniform $(K=0)$. In panel (b), just below, the arrow labeled $\mathbf{m}$ reiterates the information conveyed by the diffusing spins on the left side of panel (a). Namely, the spin accumulation in the nonmagnet is everywhere anti-parallel to $\mathbf{M}$. To understand this, we note first that $Q_{z z}^{\mathrm{FM}}>0$ on account of the first term in (10) ${ }^{20}$ Therefore, to satisfy (15), there must be a collection of spins in the non-magnet (polarized parallel to $\mathbf{M}$ ) flowing from left to right toward the interface. We interpret this, equivalently and consistently with the diffusive solutions (20) graphed as solid curves in panel (c), as a flow of anti-parallel spins away from the interface into the non-magnet. Qualitatively, we imagine that spins diffuse into the non-magnet after being "imprinted" in the anti-parallel direction by the ferromagnet. Randomly walking spins (including those that happen to diffuse back to the interface) only encounter spins parallel to the themselves because all imprinting was done by ferromagnetic spins that are parallel. From (14) and (16), $\mathbf{N}_{\mathrm{st}}=A \mathbf{Q}_{\perp^{\prime} z}^{\mathrm{NM}}=0$ because $\mathbf{m}_{\perp^{\prime}}=0$. There is no driving force for precession when the ferromagnetic magnetization is uniform. On the other hand, the spin currents $Q_{z z}^{\mathrm{NM}}$ and $\mathbf{Q}_{\perp z}^{\mathrm{NM}}$ are both non-zero [solid curves in panel (d)] because $\mathbf{M}$ is not normal to the interface.

The situation changes markedly when the magnetization $\mathbf{M}$ varies along the interface $(K \neq 0)$ [right side of Figure[2(f)]. As before, spins diffuse into the non-magnet after being imprinted in the direction anti-parallel to the local ferromagnetic magnetization. However, because the imprinting ferromagnetic spins now differ in their transverse directions, spins diffusing in the non-magnet typically encounter spins whose transverse component differs from their own. The net effect is a reduction in the transverse spin accumulation [dashed curve in $\operatorname{panel}(\mathrm{c})$ ]. The effect is greater farther away from the interface because 

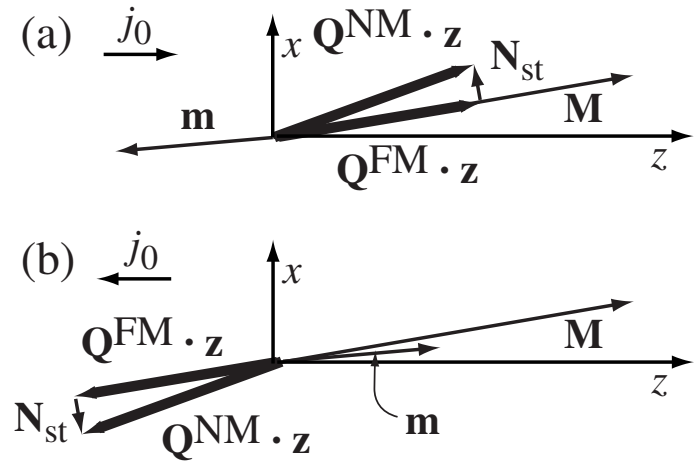

FIG. 3: (a) Interfacial spin current and spin for current flow from the non-magnet into the ferromagnet. This diagram is the same as Figure 2(e); (b) Same as (a) except that the current flows from the ferromagnet into the non-magnet.

the transverse diffusion is superimposed on longitudinal diffusion away from the interface. From (4), there is a corresponding increase in the transverse spin current near the interface [dashed curve in panel (d)]. Compared to panel (b), the spin accumulation vector $\mathbf{m}$ in panel (e) bends closer the negative $z$-axis and thus acquires a positive component perpendicular to $\mathbf{M}$. Therefore, from (14) and (16), the vectors $\mathbf{Q}^{\mathrm{NM}}$ and $\mathbf{N}_{\text {st }}$ appear as sketched in panel (e). The indicated torque tends to rotate $\mathbf{M}$ away from the interface normal. That is, $\mathbf{N}_{\mathrm{st}}$ tends to increase the amplitude $\mathbf{u}$ of the incipient spin wave assumed at the beginning of the discussion. This is the fundamental origin of the precessional instability. Thus, in contrast to the common spin-valve geometry, we have here a single ferromagnet that both generates a spin current and is acted on by that spin current.

Two additional qualitative points emerge form the discussion above. First, if the current $j_{0}$ reverses direction, so electrons flow from the ferromagnet into the nonmagnet, (10) tells us that the sign of $Q_{z z}$ reverses ${ }^{20}$ The direction of the spin accumulation reverses also so (4) remains correct. The decrease in transverse spin accumulation which rotates $\mathbf{m}$ toward the $z$-axis now implies that the direction of $\mathbf{N}_{\mathrm{st}}$ reverses as well (Figure 3). This torque tends to reduce the amplitude of the presumed spin wave. We conclude that electron flow from a ferromagnet into a non-magnet tends to suppress a precessional instability. This type of non-reciprocal behavior with respect to the direction of current flow is a characteristic feature of spin-transfer phenomena ${ }^{4}$

Finally, we remark that the presence of a second "polarizer" ferromagnetic film upstream in Figure 2 tends to enhance the precessional instability. Even the $K=0$ mode can become unstable as long as the magnetization of the polarizer is not collinear with the magnetization of the "analyzer". This is so because, in that case, the electrons incident on the $z=0$ plane carry a spin current with a non-zero component in a direction perpendicular to $\mathbf{M}$. As a practical matter, this means that the criti- cal current for the onset of precession is lower when two ferromagnets are present than for the case of a single ferromagnet studied by us.

\section{BOUNDARY CONDITIONS}

A quantitative solution of our problem requires boundary conditions for the transport and magnetization equations set down in Section II. One of these arises because the exchange interaction is effective right up to the surface of a bounded ferromagnet. For the thin film geometries of Figure 1 we show in Appendix A that an exchange torque density $\mathbf{n}_{\mathrm{ex}}=-\gamma \mathbf{M} \times \mathbf{H}^{\mathrm{S}}$ exists where

$$
\mathbf{H}_{\mathrm{ex}}^{\mathrm{S}}=\frac{\omega_{0} l_{\mathrm{ex}}^{2}}{\gamma m_{\mathrm{S}}}[\delta(z)-\delta(z-t)] \partial_{z} \mathbf{M}
$$

This shows that a localized torque acts on each interface in Figure 1(b). For the single interface geometry, the $\delta(z-t)$ term is absent. Spin wave calculations for bounded ferromagnets ${ }^{21}$ eliminate the surface torque produced by (21) by imposing the boundary condition $\partial_{z} \mathbf{M}=0$ at each interface. For our problem, the spintransfer torque (14) also acts at each interface. This dictates that we determine the spin wave modes by imposing a zero net torque requirement at each interface $\stackrel{14}{\underline{14}}$ The specific boundary condition is

$$
\mathbf{N}_{\mathrm{ex}}+\mathbf{N}_{\mathrm{st}}=0 .
$$

where $\mathbf{N}_{\text {ex }}$ is the exchange torque density $\mathbf{n}_{\text {ex }}$ integrated over an infinitesimally thin pillbox as in (14).

A distribution of charge/spin current where the spins precess at a complex frequency $\omega=\omega_{\mathrm{R}}+i \omega_{\mathrm{I}}$ is a mode of the system if (11) is satisfied in non-magnetic regions, (12) is satisfied in ferromagnetic regions, and (22) is satisfied at every interface. When $\omega_{\mathrm{I}}>0$, the mode is stable and uninteresting because its amplitude never grows large enough to be observed. True current-induced precession corresponds to modes with $\omega_{\mathrm{I}}<0$.

Of course, the currents must also be consistent with the spin-dependent scattering that occurs at the interface between a magnet and a non-magnet. Previously, two of the present authors 10 addressed this issue by imposing scattering boundary conditions on a $2 \times 2$ matrix Boltzmann equation and computing all observables from the semi-classical distribution functions $g_{\uparrow}(\mathbf{k}, \mathbf{r})$ and $g_{\downarrow}(\mathbf{k}, \mathbf{r})$. In this paper, we apply boundary conditions to the observables directly in a manner that is consistent with this Boltzmann equation treatment.

We show in Appendix B that the boundary conditions obtained in this way take the form of a set of linear kinetic equations which relate the interfacial currents to the interfacial densities. Here, we quote a form that is valid for small amplitude precessional motion around $\mathbf{H}_{\text {ext }}=H \hat{z}$. That is, we assume (here and in all subsequent work) that $\mathbf{u}=\hat{\mathbf{z}}+\mathbf{u}_{\perp}$ with $u_{\perp} \ll 1$ :

$$
j_{0}=\delta n_{0}\left[w_{0}\left(X_{\uparrow}+X_{\downarrow}\right)\right]+2 \hbar^{-1} m_{z}\left[w_{0}\left(X_{\uparrow}-X_{\downarrow}\right)\right]
$$




$$
\begin{aligned}
& -2 \hbar^{-1} \delta m\left[X_{\uparrow} w_{\uparrow}-X_{\downarrow} w_{\downarrow}\right] \\
Q_{x z}^{\mathrm{NM}}= & u_{x} Q_{z z}^{\mathrm{NM}}+w_{0}\left(m_{x}-u_{x} m_{z}\right) \\
Q_{y z}^{\mathrm{NM}}= & u_{y} Q_{z z}^{\mathrm{NM}}+w_{0}\left(m_{y}-u_{y} m_{z}\right) \\
Q_{z z}^{\mathrm{NM}}= & \frac{1}{2} \hbar \delta n_{0}\left[w_{0}\left(X_{\uparrow}-X_{\downarrow}\right)\right]+m_{z}\left[w_{0}\left(X_{\uparrow}+X_{\downarrow}\right)\right] \\
& -\delta m\left[X_{\uparrow} w_{\uparrow}+X_{\downarrow} w_{\downarrow}\right] .
\end{aligned}
$$

The constant

$$
w_{0}=\frac{A_{\mathrm{FS}}}{(2 \pi)^{3} \hbar}\left[\frac{1}{2} \frac{\partial n}{\partial \mu}\right]^{-1}
$$

where $A_{\mathrm{FS}}$ is the area of the Fermi surface projected onto the interface. The constants $w_{\uparrow}$ and $w_{\downarrow}$ are defined similarly with the densities of states per spin $\partial n_{\uparrow} / \partial \mu_{\uparrow}$ and $\partial n_{\downarrow} / \partial \mu_{\downarrow}$. Finally,

$$
X_{\sigma}=\frac{1-\mathrm{R}_{\sigma}}{4 \mathrm{R}_{\sigma}},
$$

where $\mathrm{R}_{\uparrow}=\left|R_{\uparrow}\right|^{2}$ and $\mathrm{R}_{\downarrow}=\left|R_{\downarrow}\right|^{2}$ are the squares of the majority and minority electron reflection amplitudes averaged over the Fermi surface. The appearance of the interfacial charge accumulation $\delta n_{0}$ on the right side of (23) is not inconsistent with our earlier statement that $\delta n^{\mathrm{NM}}=\delta n^{\mathrm{FM}}=0$ in the bulk. There is a resistance associated with the interface whenever $\mathrm{R}_{\sigma} \neq 0$. The corresponding voltage drop may be thought of as arising from a dipole layer of charge difference $\delta n_{0}$ localized at the interface.

We note in closing that if $\mathbf{u}_{\perp}$ were zero $(\mathbf{M} \| \hat{\mathbf{z}})$, the second and third boundary conditions in (23) reduce to (16). Appendix B demonstrates that the terms proportional to $\mathbf{u}_{\perp}$ in (23) account for the fact that $\mathbf{M}$ is slightly tilted away from the interface normal.

\section{SINGLE INTERFACE GEOMETRY}

We showed in Section III that spin-transfer torque can induce a precessional instability. To discover if it actually does so requires a full solution of the coupled transport/magnetization dynamics equations with their boundary conditions. In particular, we need convenient formulae for the interfacial torques that enter (22). For the single interface geometry of Figure 1(a), a suitable precessional ansatz for the magnetization in the nonmagnet is

$$
\mathbf{m}=\left\{\operatorname{Re}\left[m_{\perp}(z) \Phi\right], \operatorname{Im}\left[m_{\perp}(z) \Phi\right], m_{z}(z)\right\}
$$

where $\Phi=e^{i(\mathbf{K} \cdot \mathbf{R}+\omega t)}$. The corresponding ansatz in the ferromagnet is

$$
\mathbf{u}=\hat{\mathbf{z}}+\mathbf{u}_{\perp}(z) \Phi,
$$

so from (5),

$$
\mathbf{M}=M(z)\left\{\operatorname{Re}\left[u_{\perp}(z) \Phi\right], \operatorname{Im}\left[u_{\perp}(z) \Phi\right], 1\right\}
$$

where

$$
M(z)=m_{\mathrm{s}}+\delta m(z) .
$$

\section{A. Interfacial Spin-Transfer Torque}

To find the spin-transfer torque, we substitute (26) and (28) into the longitudinal component of the equations of motion (11) and (12). The solutions are [cf. (20)]

$$
m_{z}(z)=m_{z} \exp \left(z / l_{\mathrm{sf}}\right)
$$

and

$$
\delta m(z)=\delta m \exp \left(-z / \bar{l}_{\mathrm{sf}}\right)
$$

with $\bar{l}_{\mathrm{sf}}^{2}=D_{z} \bar{\tau}_{\mathrm{sf}}$. These are used in the longitudinal component of (4) and (10) to get the interfacial relations

$$
Q_{z z}^{\mathrm{NM}}=-\left(D / l_{\mathrm{sf}}\right) m_{z}=Q_{z z}
$$

and

$$
Q_{z z}^{\mathrm{FM}}=\frac{1}{2} \hbar \gamma^{\mathrm{p}} j_{0}-\left(D_{z} / \bar{l}_{\mathrm{sf}}\right) \delta m=Q_{z z} .
$$

The previous two equations, together with the first and last (longitudinal) boundary conditions in (23), determine the four interfacial quantities $\delta n_{0}, m_{z}, \delta m$, and $Q_{z z}$ in terms of $j_{0}, \gamma^{\mathrm{p}}, D / l_{\mathrm{sf}}, D_{z} / \bar{l}_{\mathrm{sf}}, X_{\uparrow}, X_{\downarrow}, w_{0}, w_{\uparrow}$, and $w_{\downarrow}$. In particular, all four are proportional to $j_{0}$, and thus change sign when the current reverses as argued earlier.

Returning to (26), we choose $\mathbf{H}_{\text {ext }}=H \hat{\mathbf{z}}$, so the transverse component of (11) is solved by

$$
m_{\perp}(z)=m_{\perp} \exp (\kappa z)
$$

where $\kappa$ satisfies

$$
l_{\mathrm{sf}}^{2} \kappa^{2}=1+l_{\mathrm{sf}}^{2} K^{2}+i \tau_{\mathrm{sf}}(\omega-\gamma H)
$$

rather than the formulae quoted after (20). Using (34), the transverse component of (4) yields

$$
\mathbf{Q}_{\perp z}^{\mathrm{NM}}=-D \kappa \mathbf{m}_{\perp}
$$

while the transverse boundary conditions in (23) are

$$
\mathbf{Q}_{\perp z}^{\mathrm{NM}}-\mathbf{u}_{\perp} Q_{z z}^{\mathrm{NM}}=w_{0}\left(\mathbf{m}_{\perp}-\mathbf{u}_{\perp} m_{z}\right) .
$$

From these, we can solve for the four interfacial quantities $\mathbf{Q}_{\perp z}^{\mathrm{NM}}$ and $\mathbf{m}_{\perp}$. The spin-transfer torque follows straightforwardly from (14) when we follow Appendix B and express $\mathbf{Q}_{\perp^{\prime} z}^{\mathrm{NM}}$ in unprimed coordinates (z-axis parallel to the interface normal) rather than primed coordinates ( $\mathbf{z}^{\prime}$-axis parallel to $\mathbf{M}$ ):

$$
\frac{\mathbf{N}_{\mathrm{st}}}{A}=\mathbf{Q}_{\perp z}^{\mathrm{NM}}-\mathbf{u}_{\perp} Q_{z z}^{\mathrm{NM}}=\mathbf{u}_{\perp} \Lambda \frac{\kappa l_{\mathrm{sf}}-1}{\kappa l_{\mathrm{sf}}+\Lambda} Q_{z z} .
$$

The constant $\Lambda=w_{0} l_{\mathrm{sf}} / D$. 


\section{B. Interfacial Exchange Torque}

The torque produced by the surface exchange field (21) over an area $A$ at $z=0$ is computed by integrating the torque density $-\gamma \mathbf{M} \times \mathbf{H}_{\mathrm{ex}}^{\mathrm{S}}$ over an infinitesimally thin pillbox as in (14). The result is

$$
\frac{\mathbf{N}_{\mathrm{ex}}}{A}=-\frac{\omega_{0} l_{\mathrm{ex}}^{2}}{m_{\mathrm{s}}} \mathbf{M} \times \partial_{z} \mathbf{M}=-i \frac{\omega_{0} l_{\mathrm{ex}}^{2}}{m_{\mathrm{s}}} M^{2}(z) \mathbf{u}_{\perp}^{\prime}
$$

because

$$
\hat{\mathbf{z}} \times \mathbf{u}_{\perp}=i \mathbf{u}_{\perp}
$$

corresponds to a $90^{\circ}$ rotation of the vector $\mathbf{u}_{\perp}$. Since the function $M(z)$ in (29) is known at this point, the only unknown in (39) is $\mathbf{u}_{\perp}^{\prime}=\left.\partial_{z} \mathbf{u}_{\perp}(z)\right|_{z=0}$.

We determine $\mathbf{u}_{\perp}^{\prime}$ numerically by integrating the transverse component of the Landau-Lifshitz-Gilbert equation (12) inward from $z=\infty$ to $z=0$. The equation of interest is

$$
\begin{aligned}
0 & =\{i \omega+\alpha \omega-i \gamma H\} \mathbf{u}_{\perp} \\
& +\frac{1}{M(z)}\left\{\frac{1}{2} \hbar \gamma^{\mathrm{p}} j_{0}-D_{z} M^{\prime}(z)\right\} \mathbf{u}_{\perp}^{\prime} \\
& +i \frac{\omega_{0} M(z) l_{\mathrm{ex}}^{2}}{m_{\mathrm{s}}}\left\{\mathbf{u}_{\perp}^{\prime \prime}+2 \frac{M^{\prime}(z)}{M(z)} \mathbf{u}_{\perp}^{\prime}-K^{2} \mathbf{u}_{\perp}\right\}
\end{aligned}
$$

The first term in brackets comes from the $\dot{M}_{\alpha}$, damping, and external field terms. The second term in brackets comes from the spin-transfer torque in the bulk of the ferromagnet due to the spatial variation of the magnetization. The third term in brackets is the bulk exchange torque. On account of (31), the spin flip term $\mathbf{u}_{\perp} \delta m / \bar{\tau}_{\text {sf }}$ in (12) cancels a piece of the spin-transfer torque. To derive (41), we have used (40) repeatedly and exploited the identity

$$
\nabla^{2}(M \mathbf{u})=\mathbf{u} \nabla^{2} M+2 \nabla M \cdot \nabla \mathbf{u}+M \nabla^{2} \mathbf{u}
$$

Very far from the interface, $M(z) \rightarrow m_{\mathrm{s}}$ and (41) reduces to

$$
\begin{aligned}
0 & =\left(i \omega+\alpha \omega-i \gamma H-i \omega_{0} K^{2} l_{\mathrm{ex}}^{2}\right) \mathbf{u}_{\perp} \\
& +\frac{1}{2} \hbar \gamma^{\mathrm{p}}\left(j_{0} / m_{\mathrm{s}}\right) \mathbf{u}_{\perp}^{\prime}+i \omega_{0} l_{\mathrm{ex}}^{2} \mathbf{u}^{\prime \prime} .
\end{aligned}
$$

This equation is solved by $\mathbf{u}_{\perp}(z)=\mathbf{u}_{\perp} \exp (-p z)$ where $(\operatorname{Re} p>0)$,

$$
p l_{\mathrm{ex}}=-i \frac{\Omega}{2 \omega_{0}} \pm \frac{1}{2 \omega_{0}} \sqrt{-\Omega^{2}-4 \omega_{0} \Delta},
$$

and $\Delta=\omega-i \omega \alpha-\gamma H-\omega_{0} K^{2} l_{\mathrm{ex}}^{2}$, where

$$
\Omega=\frac{\hbar \gamma^{\mathrm{p}} j_{0}}{2 l_{\mathrm{ex}} m_{\mathrm{s}}}
$$

This solution is used to start the inward integration of (41). We note in passing that if $\alpha=K=0$ and $q=i p$, (44) simplifies to

$$
\omega(q)=\gamma H+\omega_{0} l_{\mathrm{ex}}^{2} q^{2}-\Omega l_{\mathrm{ex}} q .
$$

This is (essentially) the dispersion relation derived by Bazaliy and co-workers ${ }^{14}$ for bulk spin waves in the presence of a specified spin current. An instability occurs ( $\omega$ goes negative) when the current is large enough that $\Omega^{2}>4 \omega_{0} \gamma H$. If we take $\mu_{0} H=1 \mathrm{~T}$, this gives a charge current density $J_{0}=|e| j_{0}>10 \times 10^{9} \mathrm{~A} / \mathrm{cm}^{2}$, which is an order of magnitude larger than the interfacial instabilities we discuss below.

It is now straightforward to search for precession-type instabilities. For given values of current $j_{0}$, external magnetic field $H$, and transverse wave vector $K$, we search through complex frequency $\left(\omega=\omega_{\mathrm{R}}+i \omega_{\mathrm{I}}\right)$ space looking for modes. That is, situations where (22) is satisfied using (38) for the interfacial spin-transfer torque and (39) for the interfacial exchange torque. If $\omega_{\text {I }}<0$, the mode is linearly unstable against precession. A suitable initial guess is the zero-current surface spin wave frequency

$$
\omega_{\mathrm{s}}=\frac{\gamma H+\omega_{0} l_{\mathrm{ex}}^{2} K^{2}}{1-i \alpha} .
$$

\section{THIN FILM GEOMETRY}

As in the previous section, the search for currentinduced precessional instabilities in the thin film geometry of Figure 1(b) begins by solving the longitudinal part of the problem. The spin accumulation decays exponentially into the non-magnetic leads so at the left $(z=0)$ and right $(z=t)$ interfaces, we have the analog of (32) for the interfacial spin currents:

$$
Q_{z z}^{\mathrm{L}}=-\frac{D_{\mathrm{s}}^{\mathrm{L}}}{l_{\mathrm{sf}}^{\mathrm{L}}} m_{z}^{\mathrm{L}} \quad Q_{z z}^{\mathrm{R}}=-\frac{D_{\mathrm{s}}^{\mathrm{R}}}{l_{\mathrm{sf}}^{\mathrm{R}}} m_{z}^{\mathrm{R}}
$$

The superscript on the diffusion constants and on the spin flip lengths allows us to assign different material properties to the leads and thus to the interfaces 15

The $z$-dependences of the spin accumulation and the spin current in the ferromagnetic film are linear combinations of $\exp \left( \pm z / \bar{l}_{\mathrm{sf}}\right)$. Straightforward algebra yields the interfacial relations

$$
\begin{aligned}
Q_{z z}^{\mathrm{L}}= & \gamma^{\mathrm{p}} j_{0} \\
& -\frac{D_{z}}{\bar{l}_{\mathrm{sf}}}\left[\delta m^{\mathrm{L}} \operatorname{coth}\left(t / \bar{l}_{\mathrm{sf}}\right)-\delta m^{\mathrm{R}} \operatorname{csch}\left(t / \bar{l}_{\mathrm{sf}}\right)\right] \\
Q_{z z}^{\mathrm{R}}= & \gamma^{\mathrm{p}} j_{0} \\
& -\frac{D_{z}}{\bar{l}_{\mathrm{sf}}}\left[-\delta m^{\mathrm{R}} \operatorname{coth}\left(t / \bar{l}_{\mathrm{sf}}\right)+\delta m^{\mathrm{L}} \operatorname{csch}\left(t / \bar{l}_{\mathrm{sf}}\right)\right] .
\end{aligned}
$$

The four equations in (48) and (49), together with the two longitudinal boundary conditions in (23) at each interface, determine the eight quantities $\delta n_{0}^{\mathrm{L}}, \delta n_{0}^{\mathrm{R}}, m_{z}^{\mathrm{L}}, m_{z}^{\mathrm{R}}$, 
$\delta m^{\mathrm{R}}, \delta m^{\mathrm{L}}, Q_{z z}^{\mathrm{L}}$, and $Q_{z z}^{\mathrm{R}}$ in terms of the physical parameters of the model. Finally, because it is used in the integration of (41), we record that

$$
\begin{aligned}
M(z)= & m_{\mathrm{s}}+\delta m^{\mathrm{L}} \operatorname{csch}\left(t / \bar{l}_{\mathrm{sf}}\right) \sinh \left[(t-z) / \bar{l}_{\mathrm{sf}}\right] \\
& +\delta m^{\mathrm{R}} \operatorname{csch}\left(t / \bar{l}_{\mathrm{sf}}\right) \sinh \left(z / \bar{l}_{\mathrm{sf}}\right) .
\end{aligned}
$$

Up to an overall sign, the spin transfer and exchange torques are still given by (38) and (39). Therefore, at the right interface, we choose $\mathbf{u}_{\perp}$ arbitrarily and compute $\mathbf{u}_{\perp}^{\prime}$ so that (22) is satisfied at that interface. This provides the initial condition for the integration of (41) toward $z=$ 0 . Then, the search through complex frequency space for solutions that satisfy (22) at the left interface proceeds as described earlier. As initial guesses, we use the zerocurrent frequencies of the spin wave modes of the film:

$$
\omega_{n}=\frac{\gamma H+\omega_{0} l_{\mathrm{ex}}^{2}\left[(n \pi / t)^{2}+K^{2}\right]}{1-i \alpha} .
$$

\section{RESULTS \& DISCUSSION}

The numerical results presented in this section are based on converged solutions of the transport and magnetization equations using material parameters derived directly from experiment or from first-principle electronic structure calculations (Table $\mathbb{I}$ ).

\section{A. Single Interface Geometry}

The top panel of Figure 4 illustrates the regions of linear instability as a function of applied charge current $J_{0}$ and wave vector $K$ parallel to the interface for the single interface geometry (top panel of Figure 1). An initial state of current induced precession occurs inside each contour labeled by a choice of external magnetic field $H$. We emphasize that every contour forms a closed loop in the $J_{0}-K$ plane. That is, for a fixed value of $J_{0}$, the instability region is bounded by a lower and an upper critical wave vector and, for a fixed value of $K$, the instability region is bounded by a lower and an upper critical current density. Note also that the instability occurs only for rather small values of external magnetic field. We will see in the next subsection that thin films support precession for much larger values of $H$. In addition, it appears that the upper critical field is very sensitive to the parameters that describe the system. A systematic exploration of parameter space is prohibitive, but we note that the choices $l_{\mathrm{sf}}=1000 \mathrm{~nm}$ and $\bar{l}_{\mathrm{sf}}=15 \mathrm{~nm}$ give regions of instability for $H>2 \mathrm{~T}$.

The bottom panel of Figure 4 is the same as the top panel except that we have replaced the full transverse

\begin{tabular}{|c|c|c|}
\hline Parameter & Value & Ref. \\
\hline$\rho(\mathrm{Cu})$ & $6 \mathrm{n} \Omega \mathrm{m}$ & 22 \\
\hline$\rho_{\uparrow}$ & $40.5 \mathrm{n} \Omega \mathrm{m}$ & 22 \\
\hline$\rho_{\downarrow}$ & $109.5 \mathrm{n} \Omega \mathrm{m}$ & 22 \\
\hline$l_{\mathrm{sf}}(\mathrm{Cu})$ & $450 \mathrm{~nm}$ & 23 \\
\hline $\bar{l}_{\mathrm{sf}}(\mathrm{Co})$ & $59 \mathrm{~nm}$ & 24 \\
\hline$A R_{\uparrow}$ & $0.117 \mathrm{f} \Omega \mathrm{m}^{2}$ & 22 \\
\hline$A R_{\downarrow}$ & $0.903 \mathrm{f} \Omega \mathrm{m}^{2}$ & 22 \\
\hline$A_{\mathrm{FS}}$ & $580 \mathrm{~nm}^{-2}$ & 25 \\
\hline$\partial n / \partial \mu$ & $6.23 \mathrm{eV}^{-1} \mathrm{~nm}^{-3}$ & 26 \\
\hline$\partial n_{\uparrow} / \partial \mu_{\uparrow}$ & $8.17 \mathrm{eV}^{-1} \mathrm{~nm}^{-3}$ & 26 \\
\hline$\partial n_{\downarrow} / \partial \mu_{\downarrow}$ & $31.6 \mathrm{eV}^{-1} \mathrm{~nm}^{-3}$ & 26 \\
\hline$A_{\mathrm{ex}}$ & $2 \times 10^{-11} \mathrm{~J} / \mathrm{m}$ & 27 \\
\hline$M_{\mathrm{s}}$ & $14.46 \times 10^{5} \mathrm{~A} / \mathrm{m}$ & 28 \\
\hline$\gamma(\mathrm{Co})$ & $1.9 \times 10^{11} \mathrm{~s}^{-1} \mathrm{~T}^{-1}$ & 29 \\
\hline$\gamma(\mathrm{Cu})$ & $1.75 \times 10^{11} \mathrm{~s}^{-1} \mathrm{~T}^{-1}$ & 25 \\
\hline$\alpha$ & 0.01 & 30 \\
\hline$D$ & $4.1 \times 10^{16} \mathrm{~nm}^{2} \mathrm{~s}^{-1}$ & \\
\hline$D_{z}$ & $1.7 \times 10^{15} \mathrm{~nm}^{2} \mathrm{~s}^{-1}$ & \\
\hline$\tau_{\mathrm{sf}}(\mathrm{Cu})$ & $2.4 \times 10^{-11} \mathrm{~s}$ & \\
\hline $\bar{\tau}_{\mathrm{sf}}(\mathrm{Co})$ & $2.0 \times 10^{-12} \mathrm{~s}$ & \\
\hline$\gamma^{\mathrm{P}}$ & 0.46 & \\
\hline$\omega_{0}$ & $3.465 \times 10^{11} \mathrm{~s}^{-1}$ & \\
\hline$l_{\mathrm{ex}}$ & $3.9 \mathrm{~nm}$ & \\
\hline $2 m_{\mathrm{s}} / \hbar$ & $143.6 \mathrm{~nm}^{-3}$ & \\
\hline$w_{0}$ & $11.4 \times 10^{14} \mathrm{nms}^{-1}$ & \\
\hline$w_{\uparrow}$ & $8.7 \times 10^{14} \mathrm{nms}^{-1}$ & \\
\hline$w_{\downarrow}$ & $2.25 \times 10^{14} \mathrm{nms}^{-1}$ & \\
\hline$X_{\uparrow}$ & 1.90 & \\
\hline$X_{\downarrow}$ & 0.24 & \\
\hline
\end{tabular}
Landau-Lifshitz-Gilbert equation (41) by

$$
0=\{i \omega+\alpha \omega-i \gamma H\} \mathbf{u}_{\perp}
$$

TABLE I: Material parameters for $\mathrm{Cu} / \mathrm{Co}$. The values above the horizontal line were taken from experiment or firstprinciples calculation. The values below the line were calculated using equations given in the text, $\sigma=1 / \rho$, and the Einstein relation $e^{2} \sigma=D \partial n / \partial \mu$.

$$
\begin{aligned}
& +\frac{1}{m_{\mathrm{s}}}\left\{\frac{1}{2} \hbar \gamma^{\mathrm{p}} j_{0}-D_{z} M^{\prime}(z)\right\} \mathbf{u}_{\perp}^{\prime} \\
& +i \omega_{0} l_{\mathrm{ex}}^{2}\left\{\mathbf{u}_{\perp}^{\prime \prime}-K^{2} \mathbf{u}_{\perp}\right\}
\end{aligned}
$$

This approximation neglects the ferromagnetic spin accumulation $\operatorname{\delta m}(z)$ in the exchange interaction and retains only its gradient variation in the bulk spin-transfer torque. Evidently, these omissions make no difference to the qualitative features of the diagram.

According to Figure 4] no precession occurs if either the current density, the magnetic field, or the parallel wave vector gets too large. We expect the instability to disappear when the Gilbert damping overwhelms the effect of the spin transfer torque. Therefore, since the damping parameter $\alpha$ enters the theory only in the combination $\alpha \omega$ [see (52)], we anticipate that the mode frequency is an increasing function of $j, H$, and $K$. 

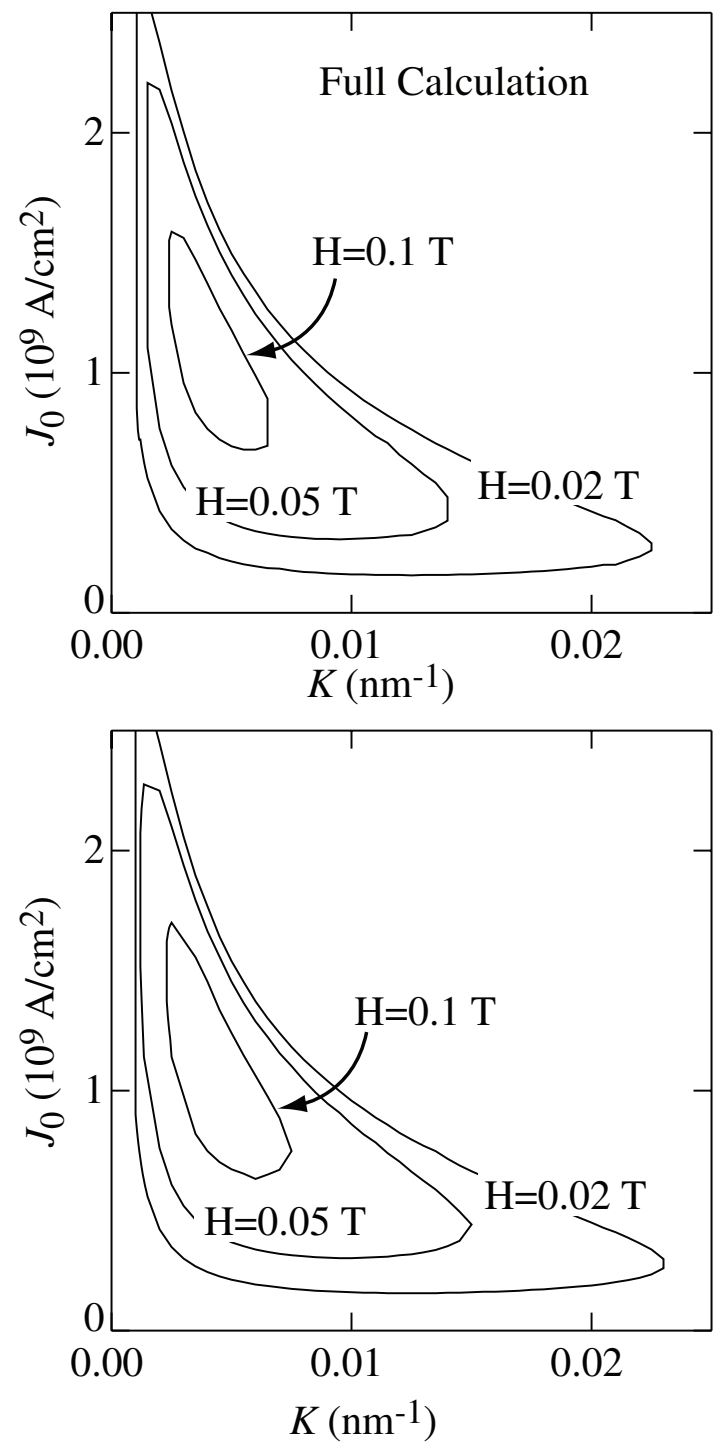

FIG. 4: Regions of instability for the single interface geometry of Figure 1(a). Each contour gives the region in $J_{0}-K$ space within which instabilities occur for an series of applied fields (labeled on each contour). The top panel gives the results for the full calculation in the ferromagnet. The bottom panel gives the results if the effect of spin accumulation on the exchange interaction is ignored.

To check this, we approximate the spin current in the second line of (52) by its value at the interface to get

$$
\begin{aligned}
0 & =\{i \omega+\alpha \omega-i \gamma H\} \mathbf{u}_{\perp} \\
& +\frac{1}{m_{\mathrm{s}}}\left\{\frac{1}{2} \hbar \gamma^{\mathrm{p}} j_{0}-D_{z} \delta m(0) / \bar{l}_{\mathrm{sf}}\right\} \mathbf{u}_{\perp}^{\prime} \\
& +i \omega_{0} l_{\mathrm{ex}}^{2}\left\{\mathbf{u}_{\perp}^{\prime \prime}-K^{2} \mathbf{u}_{\perp}\right\}
\end{aligned}
$$

This linear equation, like (43), is solved by $\mathbf{u}_{\perp}(z)=$ $\mathbf{u}_{\perp} \exp (-p z)$. Therefore,

$$
\frac{u_{\perp}^{\prime}(0)}{u_{\perp}(0)}=-p
$$

with $p$ given by (44) but with (45) replaced by

$$
\Omega=\frac{\hbar \gamma^{\mathrm{p}} j_{0} / 2-D_{z} \delta m(0) / \bar{l}_{\mathrm{sf}}}{l_{\mathrm{ex}} m_{s}}=\frac{\hbar \gamma^{\mathrm{p}} j_{0}}{2 l_{\mathrm{ex}} m_{s}} .
$$

On the other hand, replacing $\kappa$ by $K$ in (38) (large $K$ approximation) and $M(0)$ by $m_{s}$ in (39), the mode condition (22) gives

$$
\frac{u_{\perp}^{\prime}(0)}{u_{\perp}(0)}=-i \frac{Q_{z z} \Lambda}{\omega_{0} l_{\mathrm{ex}}^{2} m_{s}} \frac{K l_{\mathrm{sf}}-1}{K l_{\mathrm{sf}}+\Lambda}=-i \frac{Q_{z z} \Lambda^{\prime}}{\omega_{0} l_{\mathrm{ex}}^{2} m_{s}} .
$$

Equating (54) to (56) gives an estimate of the spin wave mode frequency $\omega=\omega_{\mathrm{R}}+i \omega_{\mathrm{I}}$. We find

$$
\begin{aligned}
\omega_{\mathrm{I}} & =\alpha \omega_{\mathrm{R}} \\
\omega_{\mathrm{R}} & =\left\{\frac{j_{0}^{2}}{\omega_{0} l_{\mathrm{ex}}^{2} m_{s}^{2}}\left[\gamma^{\prime \mathrm{p}} \Lambda^{\prime}\left(\frac{Q_{z z}}{j_{0}}\right)+\Lambda^{\prime 2}\left(\frac{Q_{z z}}{j_{0}}\right)^{2}\right]\right. \\
& \left.+\omega_{0} l_{e x}^{2} K^{2}+\gamma H\right\} \times \frac{1}{1+\alpha^{2}} .
\end{aligned}
$$

The second line in (57) confirms our expectation about the behavior of the mode frequencies and thus rationalizes the topology of Figure 4 Moreover, this analytic formula for $\omega_{\mathrm{R}}$ agrees quantitatively with the real part of the mode frequencies we find from our numerical solution of the full equations of motion. Unfortunately, (57) also predicts $\omega_{I}>0$. That is, (531) predicts only stable spin waves. These modes are unstable in the full solution of (52), but only barely so $\left(\omega_{\mathrm{I}}\right.$ is negative but very small in magnitude). In fact, the stability or instability of a given mode turns out to be an extremely sensitive function of the phase of $u_{\perp}^{\prime}(0) / u_{\perp}(0)$ and the factor $M^{\prime}(z)$ in (52). The latter is determined by the ferromagnetic spin flip length $\bar{l}_{\mathrm{sf}}$. We conclude that variation of the magnetization with the longitudinal variable $z$ is an essential ingredient for instability to occur in the single-interface geometry.

Figure 5 shows the calculated field dependence of the critical frequency $\omega_{\text {crit }}$, the critical transverse wave vector $K_{\text {crit }}$, and the critical current density $J_{\text {crit }}$, where precession first occurs in the single interface case. Consistent with (57), all three are finite as $H \rightarrow 0$. Otherwise, $\omega_{\text {crit }}$ and $J_{\text {crit }}$ vary roughly linearly with field, as they do in experiments performed on single films, ${ }^{7}$ until the instability shuts off for large enough $H$. The order of magnitude of the critical current $\left(\approx 10^{9} \mathrm{~A} / \mathrm{cm}^{2}\right)$ is also consistent with the single film experiments (and roughly ten times larger than the critical current seen in the trilayer pillars studied in Ref. 1). Note, finally, that $\omega_{\text {crit }}$ deviates systematically from the Larmor frequency $\gamma H$. 

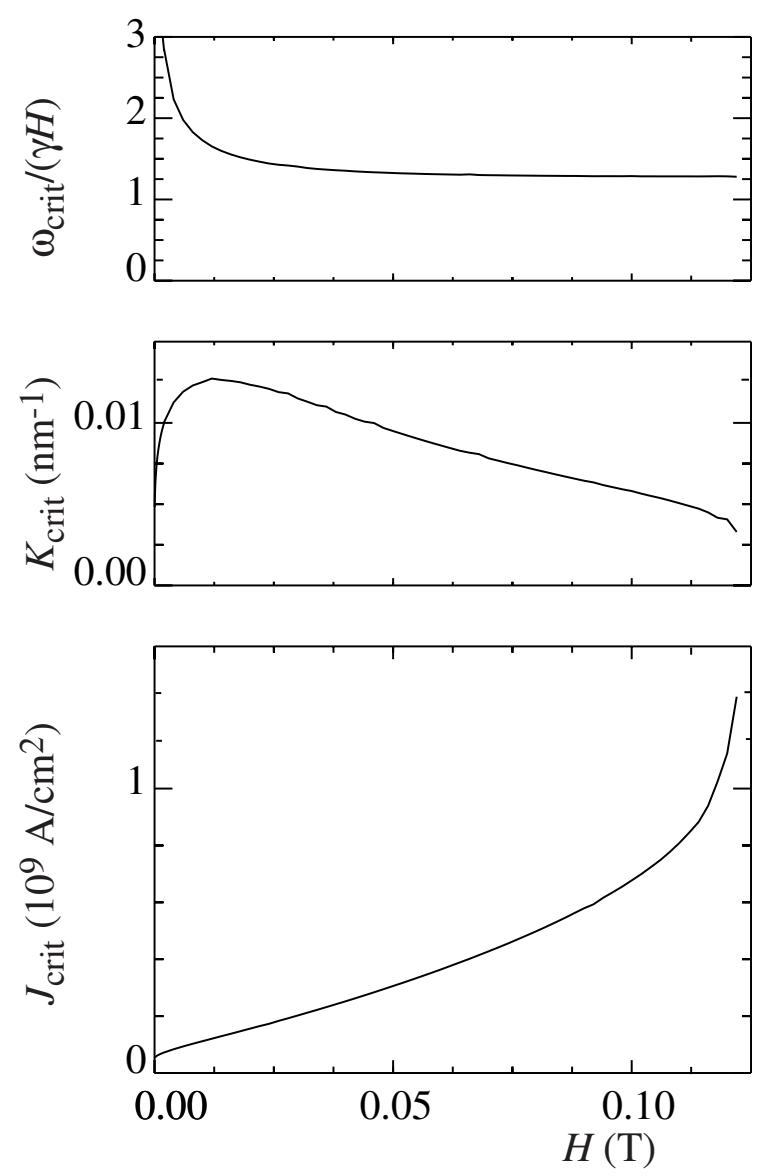

FIG. 5: Dependence on external magnetic field $H$ of the critical precession frequency $\omega_{\text {crit }}$ divided by the Larmor frequency (top panel); the critical parallel wave vector $K_{\text {crit }}$ (middle panel); and the critical current density $J_{\text {crit }}$ (bottom panel) for the single interface geometry of Figure 1a).

\section{B. Thin Film Geometry}

We remarked at the end of Section III that the interfacial spin torque associated with current flow from a ferromagnet to a non-magnet is opposite in direction to the interfacial spin torque associated with current flow from a non-magnet to a ferromagnet. As pointed out by Polianski and Brouwer, 15 this implies that zero net spin torque acts on a uniformly magnetized film attached to identical leads at identical interfaces. Those authors used leads of different lengths to break the mirror symmetry of this geometry. We discuss that case in more detail in the next section.

In our calculations for a ferromagnetic film sandwiched between two semi-infinite, non-magnetic leads (middle panel of Figure (1), the natural spatial variation of the magnetization of the spin wave modes breaks the longitudinal symmetry. Additional spatial variation arises because there is current-induced mixing amongst the modes (Figure 6). As in the single-interface problem, the spatial variation $M(z)$ is essential for precession if we assume a

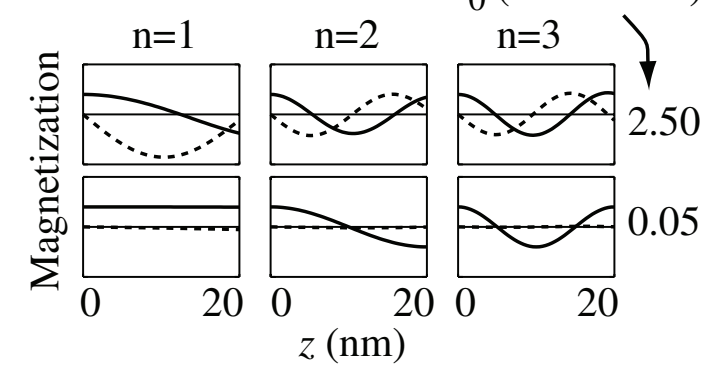

FIG. 6: Current dependence of the spatial variation of the magnetization for the three lowest spin wave modes of the ferromagnetic thin film shown in the middle panel of Figure 1 Solid curves are $M_{\mathrm{x}}(z)$. Dashed curves are $M_{\mathrm{y}}(z)$. There is almost no mode mixing for $J_{0}=0.05 \times 10^{9} \mathrm{~A} / \mathrm{cm}^{2}$.

symmetrical geometry where the scattering properties of the two interfaces are identical. We do this here, even though the formulae given in Section $\mathbf{V 1}$ allow for a more general, asymmetrical situation.

Figure 7 illustrates precessional instability diagrams for a ferromagnetic film of thickness $t=40 \mathrm{~nm}$. For each value of external magnetic field, the integer that labels each tear drop shaped region indicates the mode that goes unstable in that region. Notice that the area of the envelope in the $J_{0}-K$ plane that encloses all the $H=0.05 \mathrm{~T}$ teardrops is much larger than the area of the $H=0.05 \mathrm{~T}$ instability region for the single-interface problem sketched in Figure 4. The spin transfer torque is roughly the same in the two cases, but it is much easier to excite spin waves in the film because there is much less ferromagnetic "mass" present to contribute to the damping.

The field dependence of the critical current $J_{\text {crit }}$ is shown in Figure 8 for several film thicknesses. A relatively large current density is needed to excite the $10 \mathrm{~nm}$ film because the symmetry breaking required to generate a net torque occurs only when the lowest frequency spin wave mode (which has uniform magnetization in the $z$-direction) is perturbed by current-induced mixing with the next-highest spin wave mode (which has non-uniform magnetization in the $z$-direction). This is shown by the leftmost panel of Figure 6 We will use (55) as the characteristic frequency associated with the driving current and (51) to estimate the frequency splitting between the $n=1$ and $n=2$ film modes. Setting these equal, we get a field-independent estimate of $J_{\text {crit }}$ of the order of $10^{9}-10^{10} \mathrm{~A} / \mathrm{m}^{2}$.

The critical current density decreases in Figure 8 as the film thickness increases to $20 \mathrm{~nm}$ and then to $40 \mathrm{~nm}$ because the mode splitting decreases in typical particlein-a-box fashion. We will refer to this below as a quantum size effect. Then, beginning with the $40 \mathrm{~nm}$ film, a new phenomenon occurs. For large enough $H$, the perturbed $n=1$ precession mode never goes unstable (there is no tear drop labelled " 1 " in the $H=2 \mathrm{~T}$ panel of Figure 7). 


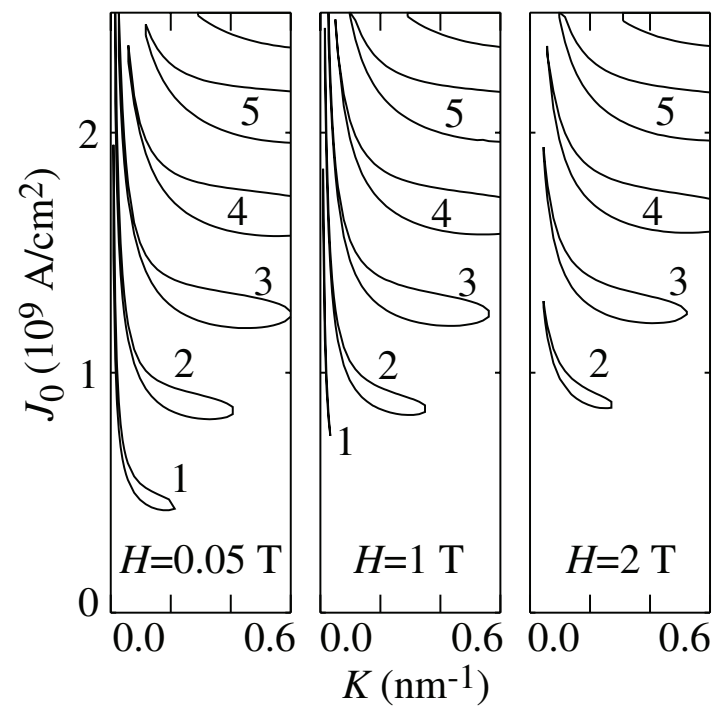

FIG. 7: Instability diagram for a $t=40 \mathrm{~nm}$ ferromagnetic film in the geometry of Figure 1(b) for three values of external magnetic field. The integers label the thin film precessional mode that goes unstable in each region.

Instead, an upward tick in $J_{\text {crit }}$ occurs when the perturbed $n=2$ spin wave mode begins to precess. For the $80 \mathrm{~nm}$ film, this phenomenon moves to lower values of $H$ and successively higher spin wave modes become the first to become unstable. This transition of instability from one mode to the next highest is responsible for the jagged appearance of the $J_{\text {crit }}(H)$ curves for the thicker films.

The nearly vertical line segment in Figure 8 is the $t=\infty$ single-interface result redrawn from the lowest panel of Figure [ 5 It is smooth because the region of instability for each mode becomes increasing small (as a function of $H$ ) until, in the limit of a semi-infinite film, every value of $H$ leads to the linear instability of a different precession mode of the film. This curve terminates at a critical field $H_{\text {crit }}(t=\infty)$. The $120 \mathrm{~nm}$ film curve shows that $H_{\text {crit }}(t)$ increases as the film thickness decreases from $t=\infty$.

Finally, Figure 9] summarizes the thickness dependence of $J_{\text {crit }}$ for three values of external magnetic field. For $H=0.1 \mathrm{~T}$, the critical current decreases monotonically as $t$ increases as the quantum size effect argument suggests. However, to reach the $t \rightarrow \infty$ limit (horizontal arrow), $J_{\text {crit }}$ must eventually increase. As the $H=1 \mathrm{~T}$ and $H=3$ T curves show, this occurs in jumps each time a mode becomes absolutely stable and the next highest mode initiates the precession. $J_{\text {crit }}(t)$ oscillates in this regime because the quantum size effect operates as long as one particular mode is responsible for the instability.

The $H=1 \mathrm{~T}$ and $H=3 \mathrm{~T}$ curves in Figure 9 terminate at a critical thickness $t_{\text {crit }}(H)$ that decreases with increasing magnetic field. This is consistent with the fact that the semi-infinite ferromagnet only supports currentinduced precession for relatively small values of external

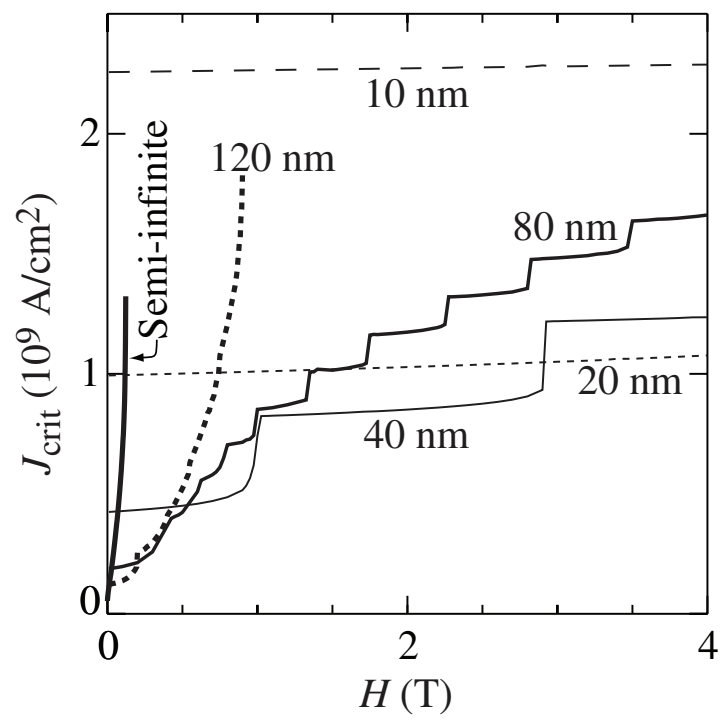

FIG. 8: Critical current as a function of field for several thicknesses of the ferromagnetic film in the geometry of Figure 1(b). The non-smooth behavior of the curves for the thicker films is associated with transitions between instabilities associated with different thin film modes.

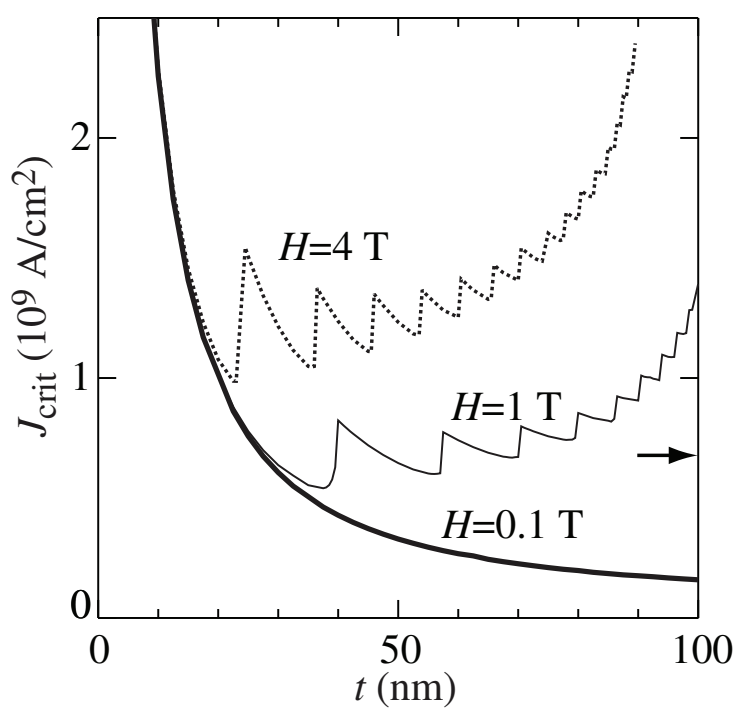

FIG. 9: Critical current as a function of ferromagnetic film thickness for three values of external magnetic field in the geometry of Figure 1(b). A jump occurs whenever a new mode is responsible for the precession. The horizontal arrow is the $H=0.1 \mathrm{~T}$ critical current for a semi-infinite ferromagnetic film.

magnetic field.

\section{EFFECT OF FINITE LEADS}

Polianski and Brouwer ${ }^{15}$ studied current-induced magnetization precession for the geometry depicted in the lowest panel of Figure 1] The unequal length leads 
that break the symmetry of the problem are each attached to an electron "reservoir". By definition, the electrochemical potential takes its equilibrium value inside each reservoir. Therefore, the spin accumulation (a nonequilibrium property) must go smoothly to zero when we enter each reservoir from the adjacent lead. These authors also fixed the magnetization of the ferromagnetic film to be uniform in the $z$ direction.

In this section, we study this reservoir geometry using our formalism, both with and without the additional restriction to uniform longitudinal magnetization in the ferromagnet. Our expectation is that the approximations of Ref. 15 will be adequate if the ferromagnetic film thickness $t$ is less than the exchange length $l_{\mathrm{ex}}$.

Let the non-magnetic leads have lengths $t^{\mathrm{L}}$ and $t^{\mathrm{R}}$ as shown in Figure 1(c). It is straightforward to check that the solution of (11) in, say, the right lead that satisfies the reservoir boundary condition of zero longitudinal and transverse spin accumulation at $z=t+t^{\mathrm{R}}$ gives at the interface

$$
\begin{aligned}
& m_{z}(t)=m_{z}^{+}\left(\exp \left[-t^{\mathrm{R}} / l_{\mathrm{sf}}\right]-\exp \left[t^{\mathrm{R}} / l_{\mathrm{sf}}\right]\right) \\
& m_{\perp}(t)=m_{\perp}^{+}\left(\exp \left[-\kappa t^{\mathrm{R}}\right]-\exp \left[\kappa t^{\mathrm{R}}\right]\right)
\end{aligned}
$$

The corresponding spin current (4) is

$$
\begin{aligned}
Q_{z z}(t) & =\left(D m_{z}^{+} / l_{\mathrm{sf}}\right)\left(\exp \left[-t^{\mathrm{R}} / l_{\mathrm{sf}}\right]+\exp \left[t^{\mathrm{R}} / l_{\mathrm{sf}}\right]\right) \\
Q_{\perp z}(t) & =\left(\kappa D m_{\perp}^{+}\right)\left(\exp \left[-\kappa t^{\mathrm{R}}\right]+\exp \left[\kappa t^{\mathrm{R}}\right]\right)
\end{aligned}
$$

These can be recast as a relation between the spin accumulation and the spin current at the interface:

$$
\begin{aligned}
Q_{z z}(t) & =-\frac{D}{l_{\mathrm{sf}}} m_{z}(t) \operatorname{coth}\left(t^{\mathrm{R}} / l_{\mathrm{sf}}\right) \\
Q_{\perp z}(t) & =-\kappa D m_{\perp}(t) \operatorname{coth}\left(\kappa t^{\mathrm{R}}\right) .
\end{aligned}
$$

These formulae are the same as those used previously except for multiplicative factors depending on the length of the leads. We recover (32) and (36) in the limit $t^{\mathrm{R}} \rightarrow \infty$. Very similar results apply to the left lead and interface.

The modified spin currents (60) can be inserted into the solution of the longitudinal spin accumulation and then the boundary conditions for the interfacial spin torque. Wherever $D$ appears explicitly (i.e. $l_{\text {sf }}$ and $\kappa$ are not affected) it can be replaced by $D$ times the factor given above. Otherwise, the full calculation for the reservoir geometry proceeds as outlined in previous sections. Figure 10 illustrates results for two films with $t^{\mathrm{L}}=\infty$. The $t^{\mathrm{R}}=450 \mathrm{~nm}$ is weakly lead asymmetric in the sense that the factor $\operatorname{coth}\left(t^{\mathrm{R}} / l_{\mathrm{sf}}\right)$ in (60) takes the value 1.3. The $t^{\mathrm{R}}=45 \mathrm{~nm}$ film is strongly lead asymmetric because $\operatorname{coth}\left(t^{\mathrm{R}} / l_{\mathrm{sf}}\right)$ has the value 10 .

From relatively small values near $t=0$ (see below), $J_{\text {crit }}$ in Figure 10 increases initially as the film thickens due to increased damping from the additional ferromagnetic material. This trend continues until the lowest mode of uniform magnetization becomes absolutely

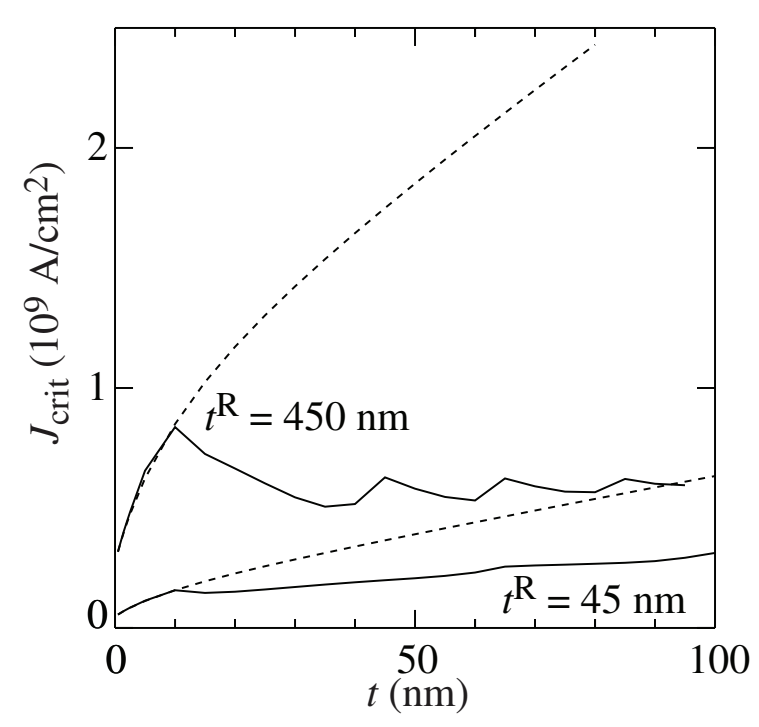

FIG. 10: Critical current as a function of ferromagnetic film thickness in the asymmetrical geometry of Figure 1(c) with $t^{\mathrm{L}}=\infty$. The dashed curves are a uniform magnetization approximation. The solid curves are the results of the full calculation. The breaks in the solid curves correspond to the onset of precession from successively higher film modes. The external field is $H=3 \mathrm{~T}$.

stable and the next higher spin wave mode initiates precession. Thereafter, the $t$ dependence of the critical current density is determined by a competition between the quantum size effect (which tends to decrease $J_{\text {crit }}$ at $t$ increases) and increased damping (which tends to increase $J_{\text {crit }}$ as $t$ increases).

A comparison of Figure 9 with Figure 10 shows that the very thin film limit of $J_{\text {crit }}$ depends very sensitively on the asymmetry of the leads. When the leads have equal length (Figure 9), the spin current and torque come exclusively from the $D_{z} M^{\prime}(z)$ term in (41). This implies a large critical current density because a large amount of energy is needed to mix the modes when $t \rightarrow 0$ [see (51)]. By contrast, the critical current density is relatively small when the leads have unequal length (Figure 10) because (even if the magnetization is uniform) there is incomplete cancellation between the (oppositely signed) spin transfer torques generated at the two ends of the ferromagnet. 15 Figure [1] shows that $J_{\text {crit }}$ actually increases exponentially with $t^{\mathrm{R}}$ when $t^{\mathrm{L}}=\infty$.

We now consider an approximation to these full calculations that forces the magnetization in the ferromagnetic film to be uniform in the $z$ direction. This occurs automatically in our numerical work if the exchange length $l_{\text {ex }}$ and the spin diffusion length $\bar{l}_{\text {sf }}$ are made much larger than any thickness $t$ of interest. The results are shown as dashed curves in Figure 10. As expected, the full and approximate calculations agree quantitatively only when $t$ is smaller than or approximately equal to the true Co exchange length of $3.9 \mathrm{~nm}$ (see Table I). The agreement for $t=10 \mathrm{~nm}$ is somewhat accidental because (as the break 


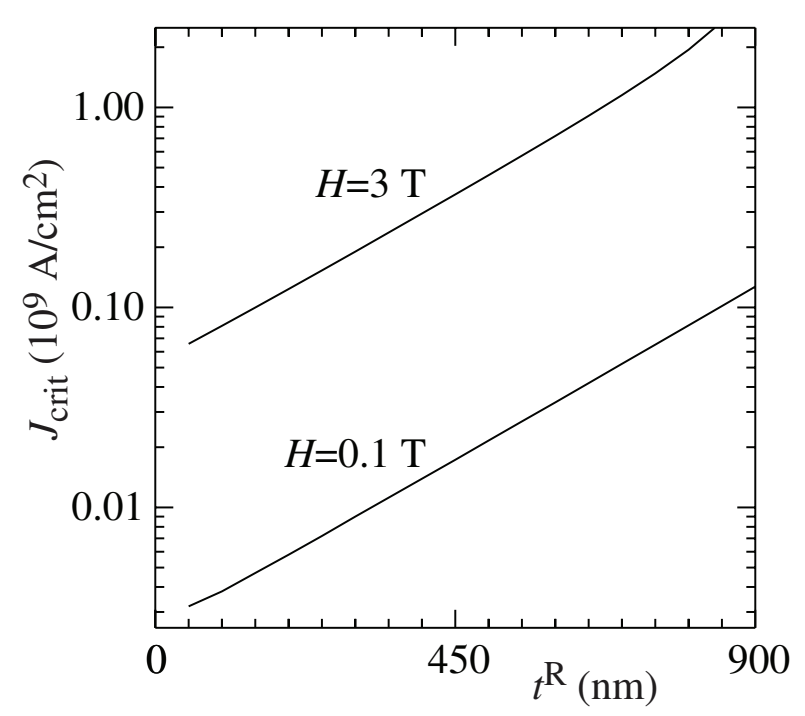

FIG. 11: Log-linear plot of critical current in the geometry of Figure 1(c) as a function of $t^{\mathrm{R}}$ when $t^{\mathrm{L}}=\infty$. The thickness of the ferromagnetic film is $t=2.5 \mathrm{~nm}$.

in the curve indicates) the precession at this thickness is not associated with a state of uniform magnetization in the longitudinal direction.

On the other hand, Figure 10 shows that for our choice of parameters, the uniform magnetization approximation gets relatively better (for $t \gg l_{\mathrm{ex}}$ ) as the lengths of the two leads become more dissimilar. Qualitatively, this occurs because there are two sources of net spin transfer torque in this problem: the longitudinal asymmetry of the leads and the longitudinal asymmetry of the magnetization. If the first dominates the second (strong lead asymmetry), it does not really matter which mode goes unstable and the uniform magnetization approximation will be acceptable. If the second dominates the second (weak lead asymmetry), the mode identity is crucial and the approximate calculation fails badly.

\section{LIMITATIONS \& FUTURE DIRECTIONS}

In experiment, the large current densities required to produce current-induced precession are achieved using point-contact, nanowire, or nanopillar geometries where conductor dimensions in the direction perpendicular to the current flow are "necked-down" from micron-scale dimensions to hundred nanometer-scale dimensions. The reservoir geometry of Figure 1(c) is an approximate way to model this behavior within the context of a strictly one-dimensional calculation. Nonetheless, it remains true that we have ignored the fact that the current only acts over a small area. The small area is either a small part of a large film, or a small finite element. In both cases, we have ignored the behavior of the current at the edges.

For a point contact geometry, we have ignored how the current spreads into the film, how the variation in the magnetization decays into the film both laterally and in the interface direction, and how these interact. For a nanowire or nanopillar geometry, we have ignored the behavior of the current at the edges. Clearly, these threedimensional effects will need to be considered if calculations of the sort presented here are ever to be truly predictive in the sense of device design.

Our calculations take no account of magnetostatics. The necessary computations are a bit complex because the magnetic self-energy is a non-local function of the spin density. It is true that the dominant effect can be modelled as magnetic anisotropy (which we have also ignored), but the known effects of magnetostatics on spinwave dispersion suggest the possibility of other effects for the present problem.

More significantly, we have focused exclusively on the initial, linear instability of a current-carrying nanomagnet toward precession. In reality, non-linear effects cut off the exponential growth of the mode amplitudes we find. This means that the final magnetic dynamical state may have little in common with simple precession, particularly for magnets whose size significantly exceeds an exchange length. To our knowledge, little or nothing is known about spatially inhomogeneous magnetic states of this type in the present context.

Finally, we have performed all of our calculations at zero temperature. An extension to finite temperature clearly would be interesting in light of recent experiments on thermally activated magnetic reversal induced by a spin-polarized current $\underline{\underline{31}}$

\section{SUMMARY}

We have combined a drift-diffusion description of transport with a Landau-Lifshitz-Gilbert description of magnetization dynamics to demonstrate that unpolarized current flow from a non-magnet into a ferromagnet can produce a precession-type instability of the magnetization. Neglecting magnetic anisotropy and magnetostatics, our numerical calculations show (for both singleinterface and thin film geometries) that a linear instability occurs when both the current density and the excitation wave vector parallel to the interface are neither too small nor too large. The critical current density generally increases with increasing magnetic field until the instability is quenched. Compared to the single-interface geometry, the film geometry supports a precessional instability over a much larger range of external magnetic field. In general, a particular spin wave mode initiates the instability when the mode just below it (in energy) becomes absolutely stable against current-induced precession.

In a lead-film-lead geometry, it is necessary to break mirror symmetry to generate precession because equal and opposite spin transfer torques are generated at the two ends of the ferromagnetic film. In our model, the required symmetry breaking is provided by spin wave 
modes of the film whose magnetization varies along the direction of current flow. If the magnetization is artificially constrained to be longitudinally uniform, asymmetry must be introduced in the length or properties of the leads or in the scattering properties of the two interfaces. When the spin wave mode asymmetry generates the spintransfer torque, the critical current density first decreases (a quantum size effect) and then slowly increases (as successive modes become stable against precession) as the film thickness increases. When interface or lead asymmetry dominates the spin transfer torque, the critical current is small (compared to the mode-dominated regime) and slowly increases due to increased damping as the film thickness increases.

\section{ACKNOWLEDGMENT}

One of us (J.X.) acknowledges support from the National Science Foundation under grant DMR-9820230. The authors thank Dr. Robert McMichael for essential help with the references.

\section{Appendix A: Exchange Magnetic Field}

The total exchange energy of a semi-infinite ferromagnet that extends from $z=z_{\mathrm{L}}$ to $z=z_{\mathrm{R}}$ is

$$
E_{\mathrm{ex}}=\frac{A_{\mathrm{ex}}}{m_{\mathrm{s}}^{2}} \int d^{2} R \int_{z_{\mathrm{L}}}^{z_{\mathrm{R}}} d z\left(\partial_{\alpha} \mathbf{M}\right)\left(\partial_{\alpha} \mathbf{M}\right)
$$

The quantity $m_{\mathrm{s}}$ occurs here because $M$ denotes spin density rather than magnetization. Focus on the $\alpha=$ $z$ component and let $\mathbf{M} \rightarrow \mathbf{M}+\boldsymbol{\eta}$. This induces the variation

$$
\delta E_{\mathrm{ex}}=\frac{2 A_{\mathrm{ex}}}{m_{\mathrm{s}}^{2}} \int d^{2} R \int_{z_{\mathrm{L}}}^{z_{\mathrm{R}}} d z\left(\partial_{z} \boldsymbol{\eta}\right) \cdot\left(\partial_{z} \mathbf{M}\right) .
$$

Integrating by parts gives

$\left.\delta E_{\mathrm{ex}}=\left.\frac{-2 A_{\mathrm{ex}}}{m_{\mathrm{s}}^{2}} \int d^{2} R\left[\int_{z_{\mathrm{L}}}^{z_{\mathrm{R}}} d z \boldsymbol{\eta} \cdot \partial_{z}^{2} \mathbf{M}-\left[\boldsymbol{\eta} \cdot \partial_{z} \mathbf{M}\right)\right]\right|_{z_{\mathrm{L}}} ^{z_{\mathrm{R}}}\right]$

or

$$
\begin{aligned}
\delta E_{\mathrm{ex}}= & \frac{-2 A_{\mathrm{ex}}}{m_{\mathrm{s}}^{2}} \int d^{2} R \int_{z_{\mathrm{L}}}^{z_{\mathrm{R}}} d z \boldsymbol{\eta} \cdot\left\{\partial_{z}^{2} \mathbf{M}\right. \\
& \left.+\partial_{z} \mathbf{M}\left[\delta\left(z-z_{\mathrm{L}}\right)-\delta\left(z-z_{\mathrm{R}}\right)\right]\right\} .
\end{aligned}
$$

The exchange field $H_{\mathrm{ex}}=-\gamma^{-1}\left(\delta E_{\mathrm{ex}} / \delta \mathbf{M}\right)$. Therefore, since $\boldsymbol{\eta} \equiv \delta \mathbf{M}$,

$$
\mathbf{H}_{\mathrm{ex}}=\frac{2 A_{\mathrm{ex}}}{\gamma m_{\mathrm{s}}^{2}}\left\{\partial_{z}^{2} \mathbf{M}+\partial_{z} \mathbf{M}\left[\delta\left(z-z_{\mathrm{L}}\right)-\delta\left(z-z_{\mathrm{R}}\right)\right]\right\} .
$$

The $\alpha=x$ and $\alpha=y$ components of (61) do not generate surface terms so (65) generalizes to

$$
\begin{aligned}
\mathbf{H}_{\mathrm{ex}} & =\frac{2 A_{\mathrm{ex}}}{\gamma m_{\mathrm{s}}^{2}} \nabla^{2} \mathbf{M} \\
& +\frac{2 A_{\mathrm{ex}}}{\gamma m_{\mathrm{s}}^{2}}\left[\delta\left(z-z_{\mathrm{L}}\right)-\delta\left(z-z_{\mathrm{R}}\right)\right] \partial_{z} \mathbf{M} .
\end{aligned}
$$

\section{Appendix B: Transport Boundary Conditions}

The interfacial boundary conditions (23) for the driftdiffusion equations (2), (44), (7), and (9) can be derived most convincingly by averaging the boundary conditions for the semi-classical distribution functions $g_{\uparrow}(\mathbf{k}, \mathbf{r})$ and $g_{\downarrow}(\mathbf{k}, \mathbf{r})$ used in a Boltzmann equation treatment of spin transport ${ }^{32}$ A simpler alternative is to proceed phenomenologically and write kinetic equations that connect the currents to the densities. Temporarily, we assume that the ferromagnetic magnetization $\mathbf{M}=M \mathbf{u}$ is parallel to the interface normal, i.e., parallel to the z-axis.

In linear approximation, the longitudinal particle current that flows through the interface for each spin type is

$$
\begin{aligned}
& j_{\uparrow}=k_{\uparrow}\left(\mu_{\uparrow}^{\mathbf{N M}}-\mu_{\uparrow}^{\mathbf{F M}}\right)=\frac{1}{2} k_{\uparrow}\left(a_{0} \delta n_{\uparrow}^{\mathbf{N M}}-a_{\uparrow} \delta n_{\uparrow}^{\mathbf{F M}}\right) \\
& j_{\downarrow}=k_{\downarrow}\left(\mu_{\downarrow}^{\mathbf{N M}}-\mu_{\downarrow}^{\mathbf{F M}}\right)=\frac{1}{2} k_{\downarrow}\left(a_{0} \delta n_{\downarrow}^{\mathbf{N M}}-a_{\downarrow} \delta n_{\downarrow}^{\mathbf{F M}}\right) .
\end{aligned}
$$

Here, $k_{\uparrow}$ and $k_{\downarrow}$ are kinetic coefficients, $\mu_{\uparrow}^{\mathrm{NM}}, \mu_{\downarrow}^{\mathrm{NM}}, \mu_{\uparrow}^{\mathrm{FM}}$, and $\mu_{\downarrow}^{\mathrm{FM}}$ are electrochemical potentials, and $a_{0}, a_{\uparrow}$, and $a_{\downarrow}$ are twice the inverse density of states per spin at the Fermi level for electrons in the non-magnet and for majority and minority electrons in the ferromagnet, respectively. Using (67), the same algebra that led from (6) to (7) now yields

$$
\begin{aligned}
j & =j_{\uparrow}+j_{\downarrow} \\
& =\left(k_{\uparrow}+k_{\downarrow}\right) a_{0} \delta n^{\mathrm{NM}}-\left(a_{\uparrow} k_{\uparrow}+a_{\downarrow} k_{\downarrow}\right) \delta n^{\mathrm{FM}} \\
& +\frac{2}{\hbar}\left(k_{\uparrow}-k_{\downarrow}\right) a_{0} m_{z}-\frac{2}{\hbar}\left(a_{\uparrow} k_{\uparrow}-a_{\downarrow} k_{\downarrow}\right) \delta m .
\end{aligned}
$$

The physical charge accumulation $\delta n_{0}$ at the interface can be partitioned arbitrarily between $\delta n^{\mathrm{NM}}$ and $\delta n^{\mathrm{FM}}$. If we choose $\delta n=\delta n^{\mathrm{NM}}$ and $\delta n^{\mathrm{FM}}=0$, the boundary condition for the particle current becomes

$$
\begin{aligned}
j & =\left(k_{\uparrow}+k_{\downarrow}\right) a_{0} \delta n_{0}+2 \hbar^{-1}\left(k_{\uparrow}-k_{\downarrow}\right) a_{0} m_{z} \\
& -2 \hbar^{-1}\left(a_{\uparrow} k_{\uparrow}-a_{\downarrow} k_{\downarrow}\right) \delta m .
\end{aligned}
$$

In exactly the same way, we derive the boundary condition for the longitudinal spin current from

$$
\begin{aligned}
Q_{z z} & =j_{\uparrow}-j_{\downarrow} \\
& =\frac{1}{2} \hbar\left(k_{\uparrow}-k_{\downarrow}\right) a_{0} \delta n+\left(k_{\uparrow}+k_{\downarrow}\right) a_{0} m \\
& -\left(a_{\uparrow} k_{\uparrow}+a_{\downarrow} k_{\downarrow}\right) \delta m .
\end{aligned}
$$

Equations (69) and (70) reproduce the first and last boundary conditions in (23) if we use the Boltzmann equation treatment of the boundary conditions ${ }^{32}$ to make the choice

$$
k_{\sigma}=\frac{A_{\mathrm{FS}}}{(2 \pi)^{3} \hbar} \frac{1-\mathrm{R}_{\sigma}}{4 \mathrm{R}_{\sigma}} .
$$



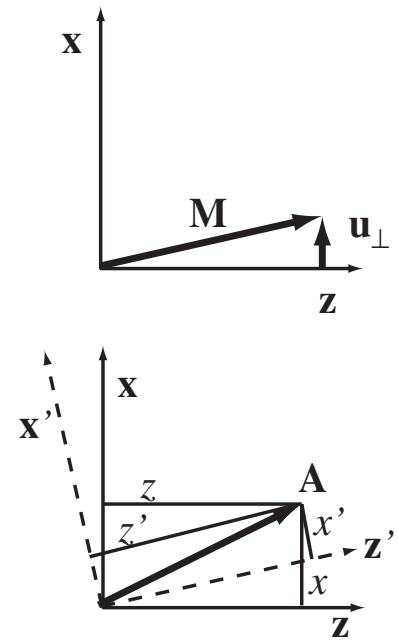

FIG. 12: The z-axis of unprimed coordinate system is perpendicular to the material interface. The z-axis of the primed coordinate system is parallel to the magnetization $\mathbf{M}$.

We stated the boundary condition for the transverse spin current in Section III, leaving a constant of proportionality $b$ unspecified in (16). As above, we choose this constant for consistency with the Boltzmann equation treatment ${ }^{32}$. The result is

$$
\mathbf{Q}_{\perp z}^{\mathrm{NM}}=\frac{A_{\mathrm{FS}}}{(2 \pi)^{3} \hbar} a_{0} \mathbf{m}_{\perp},
$$

where $A_{\mathrm{FS}}$ is the area of the Fermi surface projected onto the interface. The reflection coefficients in (71) do not appear in (72) despite the fact that spins can rotate into the transverse direction upon quantum mechanical reflection or transmission at an interface. This is so because the rotation effect disappears after Fermi surface averaging $\underline{9}$

Tserkovnyak and co-workers ${ }^{33}$ discuss another contribution to $Q_{\alpha \beta}^{\mathrm{NM}}$ due to "spin-pumping" from an adjacent ferromagnet. When the magnetization is time-harmonic with frequency $\omega$, the time derivative of the magnetization can have a component that is transverse to the magnetization and there is an additional term in (72)

$$
\mathbf{Q}_{\perp z}^{\mathrm{NM}}=\frac{A_{\mathrm{FS}}}{(2 \pi)^{3} \hbar} a_{0} \mathbf{m}_{\perp}+\frac{A_{\mathrm{FS}}}{(2 \pi)^{3}} \frac{\hbar \omega}{2} \mathbf{u}_{\perp}
$$

For our choice of parameters, this new term is numerically much smaller than the other term except for high frequencies and low currents. Nevertheless, we have retained it for all the calculations presented here.

As a final step, we must take explicit account of the fact that $\mathbf{M}$ is tilted slightly away from the interface normal $\hat{\mathbf{z}}$. This is not difficult if we define a a new coordinate system $x^{\prime} y^{\prime} z^{\prime}$ where $\hat{\mathbf{z}}^{\prime}$ points along $\mathbf{M}$ (Figure 12). In that case, for example,

$$
\mathbf{Q}_{\perp^{\prime} z}^{\mathrm{NM}}=\frac{A_{\mathrm{FS}}}{(2 \pi)^{3} \hbar} a_{0} \mathbf{m}_{\perp^{\prime}},
$$

replaces (72) as the boundary condition for the transverse spin current. However, because the direction of $\mathbf{u}_{\perp}$ in Figure 12 varies along the interface when the excitation wave vector $K \neq 0$, it behooves us to evaluate (74) using the original, interface-referenced coordinates $x y z$.

The transformation law between the $x y z$ and $x^{\prime} y^{\prime} z^{\prime}$ coordinate systems (Figure 12) simplifies considerably when $u_{\perp} \ll 1$, as is the case for our problem. Then,

$$
\begin{aligned}
A_{x}^{\prime} & =A_{x}-u_{\perp} A_{z} \\
A_{y}^{\prime} & =A_{y}-u_{\perp} A_{z} \\
A_{z}^{\prime} & =A_{z},
\end{aligned}
$$

for any vector $\mathbf{A}$ that lies close to the $z$ axis, This rule shows that (74) is indeed identical to the transverse boundary conditions stated in (23). It also shows that the longitudinal boundary conditions stated above are unaffected by the fact that $\mathbf{u}_{\perp} \neq 0$.
1 E. B. Myers, D. C. Ralph, J. A. Katine, R. N. Louie, R. A. Buhrman, Science 285, 867 (1999); J. A. Katine, F. J. Albert, R. A. Buhrman, E. B. Myers, D. C. Ralph, Phys. Rev. Lett. 84, 3149 (2000).

2 J. Grollier, V. Cros, A. Hamzic, J. M. George, H. Jaffrés, A. Fert, G. Faini, J. Ben Youssef, H. Legall, Appl. Phys. Lett. 78, 3663 (2001).

3 J.E. Wegrowe, D. Kelly, T. Truong, Ph. Guittienne, and J.-Ph. Ansermet, Europhys. Lett. 56, 748 (2001).

4 J. C. Slonczewski, J. Magn. Magn. Mater. 159, L1 (1996); J.C. Slonczewski, J. Magn. Magn. Mater. 195, L261 (1999).

5 L. Berger, Phys. Rev. B 54, 9353 (1996).

6 M. Tsoi, A. G. M. Jansen, J. Bass, W. C. Chiang, M. Seck, V. Tsoi, P. Wyder, Phys. Rev. Lett. 80, 4281 (1998); J. Z. Sun, J. Magn. Magn. Mater. 202, 157 (1999); J.E. We- grove, D. Kelly, P. Guitienne, Y. Jaccard, and J.P. Ansermet, Europhys. Lett. 45, 626 (1999).

7 Y. Ji, C.L. Chien, and M.D. Stiles, Phys. Rev. Lett. 90, 106601 (2003).

8 S.I. Kiselev, J.C. Sankey, I.N. Krivorotov, N.C. Emley, R.J. Schoelkopf, R.A. Buhrman, and D.C. Ralph cond-mat/0306259 W.H. Rippard, M.R. Pufall, S. Kaka, S.E. Russek, and T.J. Silva cond-mat/0309042 Previous experiments [M. Tsoi, A.G.M. Jansen, J. Bass, W.-C. Chiang, V. Tsoi, and P. Wyder, Nature 406, 46 (2000)] demonstrated that external microwave irradiation induces new resistance anomalies in magnetic multilayers when the current exceeds a critical value.

9 M.D. Stiles and A. Zangwill, Phys. Rev. 66, 014407 (2002).

10 M.D. Stiles and A. Zangwill, J. Appl. Phys. 91, 6812 (2002). 
11 Y. Tserkovnyak, A. Brataas, and G.E.W. Bauer, Phys. Rev. B 67, 140404 (2003).

12 Ya. B. Bazaliy, B.A. Jones, and S.-C. Zhang, cond-mat/0009034

13 J.Z. Sun, Phys. Rev. B 62, 570 (2000).

14 Ya. B. Bazaliy, B.A. Jones, and S.-C. Zhang, Phys. Rev. B 57, R3213 (1998).

15 M.L. Polianski and P.W. Brouwer, cond-mat/0304069

16 A. Brataas, Y.V. Nazarov, and G.E.W. Bauer, Eur. Phys. J. B 22, 99 (2001).

17 A paper that considers spin accumulation and spin current transverse to the ferromagnetic magnetization is A. Shpiro, P.M. Levy, and S. Zhang, Phys. Rev. B 67, 104430 (2003).

18 A. Fert, J. Phys. C 2, 1784 (1969).

19 X. Waintal, E.B. Myers, P.W. Brouwer, and D.C. Ralph, Phys. Rev. B 62, 12317 (2000).

20 The first term on the right side of (10) shows that $Q_{z z}^{\mathrm{FM}}$ is positive deep in the bulk where $\delta m=0$. The diffusive correction can change its sign near the interface, but this does not occur for the physical range of parameters we use.

21 See, for example, A.G. Gurevich and G.A. Melkov, Magnetization Oscillations and Waves, (CRC Press, Boca Raton, 1996), Chapter 7.

22 J. Bass and W. P. Pratt, J. Magn. Magn. Mater. 200, 274 (1999).

23 Q. Yang, P. Holody, S.-F. Lee, L. L. Henry, R. Loloee, P.
A. Schroeder, W. P. Pratt, Jr., and J. Bass , Phys. Rev. Lett. 72, 3274 (1994).

24 L. Piraux S. Dubois, A. Fert, and L. Belliard, Eur. Phys. J. B 4, 413 (1998).

25 N.W. Ashcroft and N.D. Mermin, Solid State Physics (Saunders, Philadelphia,1976), Table 2.1.

26 D. A. Papaconstantopoulos, Handbook of the Band Structures of Elemental Solids (Plenum, New York, 1986).

27 A range of values have been found experimentally, see for example: G. Shirane, V. J. Minkiewicz, and R. Nathans, J. App. Phys 39, 383 (1968) and Z. Frait, Phys. Stat. Solidi 2, 1417 (1969).

28 R. Pauthenet, J. Appl. Phys 538187 (1982).

29 S. M. Bhagat and P. Lubitz, Phys Rev. B 10, 179 (1974).

30 R.D.McMichael (private communication).

31 E.B. Myers, F.J. Albert, J.C. Sankey, E. Bonet, R.A. Buhrman, and D.C. Ralph, Phys. Rev. Lett. 89, 196801 (2002).

32 M.D. Stiles (unpublished).

33 Y. Tserkovnyak, A. Brataas, and G.E.W. Bauer, Phys. Rev. Lett. 88, 117601 (2002); A. Brataas, Y. Tserkovnyak, G.E.W. Bauer, and B.I. Halperin, Phys. Rev. B 66, 060404 (2002); Y. Tserkovnyak, A. Brataas, and G.E.W Bauer, Phys. Rev. B 67, 140404(R) (2003). 